\title{
Sets of orthogonal three-dimensional polarization states and their physical interpretation
}

\author{
José J. Gilø, ${ }^{1, *}$ Ignacio San José, ${ }^{2}$ Andreas Norrman, ${ }^{3}$ Ari T. Friberg, ${ }^{4}$ and Tero Setälä ${ }^{4}$ \\ ${ }^{1}$ Departamento de Física Aplicada, Universidad de Zaragoza, Pedro Cerbuna 12, 50009 Zaragoza, Spain \\ ${ }^{2}$ Instituto Aragonés de Estadística, Gobierno de Aragón, Bernardino Ramazzini 5, 50015 Zaragoza, Spain \\ ${ }^{3}$ Max Planck Institute for the Science of Light, Staudtstraße 2, D-91058 Erlangen, Germany \\ ${ }^{4}$ Institute of Photonics, University of Eastern Finland, P.O. Box 111, FI-80101 Joensuu, Finland
}

(Received 4 May 2019; published 18 September 2019)

\begin{abstract}
The spectral and characteristic decompositions of the polarization matrix provide fruitful frameworks for the physical interpretation of three-dimensional (3D) partially polarized light fields. The decompositions are formulated in terms of the three pure eigenstates, which in turn are represented through their associated orthogonal complex 3D Jones vectors. This mathematical orthogonality does not correspond, in general, to orthogonality of the polarization-ellipse planes of the respective eigenstates. Consequently, due to such inherent mathematical complexity, the geometric and physical interpretation of these sets of orthogonal complex vectors, being essential for the best understanding of the structure and properties of partially polarized 3D light, has not been addressed thoroughly. In this work, the geometric and physical features of sets of three orthonormal 3D Jones vectors are identified and analyzed, allowing one to obtain meaningful interpretations of any given mixed (partially polarized) 3D polarization state in terms of either the spectral or the characteristic decompositions. Among other results, it is found that, given a pure polarization state, any plane in space contains the polarization ellipse of a pure state that is orthogonal to it, and the mathematical expressions for the azimuth and ellipticity of such an ellipse are calculated in terms of the angular parameters determining said plane and the ellipticity of the given state. Furthermore, the spin vectors of the three polarization eigenstates are arranged in a peculiar spatial manner, such that they lie in a common plane. Beyond polarization phenomena, the approach presented also has potential applications in areas where $3 \times 3$ unitary matrices play a key role, like three-level quantum systems and gates for ternary quantum logic circuits.
\end{abstract}

DOI: 10.1103/PhysRevA.100.033824

\section{INTRODUCTION}

Polarization is a fundamental property of light and plays a vital role in understanding and exploiting electromagnetic interactions of diverse physical nature [1,2]. The polarization properties of random light fields, in their most general threedimensional (3D) representation, have lately been a subject of increasing interest within modern optical physics because of the rapid progress in nonparaxial optics and nanophotonics [3-13]. The 3D character of random light is especially encountered in the context of high numerical aperture systems [14,15], optical evanescent waves [16,17], and plasmonic surface fields $[18,19]$, highlighting the necessity to develop appropriate theoretical methods for the treatment and physical interpretation of general 3D states of polarization.

Yet, due to the mathematical complexity inherent to 3D light fields, the analysis and physical understanding of 3D polarization states are more involved than for 2D (twodimensional) states for which the polarization ellipse is restricted to a fixed plane. For instance, the Jones vectors of 2D states have a straightforward interpretation in terms of the polarization-ellipse parameters of the totally polarized component of the state. On the other hand, the concept and characterization of $3 \mathrm{D}$ polarization states rely strongly on

\footnotetext{
*ppgil@unizar.es
}

the corresponding set of orthonormal 3D Jones eigenstates $\left(\hat{\mathbf{u}}_{1}, \hat{\mathbf{u}}_{2}, \hat{\mathbf{u}}_{3}\right)$, which in general involves up to six independent parameters (plus respective arbitrary phase factors that do not affect the states of polarization). Thus, the geometric and physical interpretation of sets of orthonormal 3D Jones vectors, forming the core of this work, constitutes a key objective in polarization theory. Our results provide the polarimetric characterization of any set $\left(\hat{\mathbf{u}}_{1}, \hat{\mathbf{u}}_{2}, \hat{\mathbf{u}}_{3}\right)$ by means of well-defined and significant geometric parameters, which allows one to identify the respective polarization ellipses in meaningful geometrical terms. Furthermore, besides offering proper physical interpretations for the spectral and characteristic decompositions of any 3D polarization matrix, our work reveals some peculiar properties of the feasible spatial configurations of said eigenstates and their respective spin vectors (intrinsic angular momenta).

This paper is organized as follows. In Sec. II the necessary concepts and notations are introduced. A canonical set of orthogonal 3D Jones vectors is defined in Sec. III, which provides the mathematical basis for the general parametrization of arbitrary orthogonal sets performed in this section. Section IV is devoted to a brief analysis of the main features and peculiarities of sets of orthonormal linearly polarized states. Next, in order to illustrate the general results obtained, a pair of particularly representative families of sets of orthonormal 3D Jones vectors is analyzed in detail in Sec. V. The general results are then applied, in Sec. VI, to a 
significant physical situation of an incoherent mixture of two circularly polarized states whose polarization circles lie in mutually orthogonal planes, which, after the identification of the corresponding Jones eigenstates, turns out to be equivalent to a certain incoherent mixture of two components of the canonical set dealt with in Sec. III. Finally, the main results are summarized and briefly discussed in Sec. VII.

The original procedure for the calculation of the characteristic angular parameters of a 3D Jones vector is developed in Appendix A, while Appendix B deals with the demonstration that, given a pure polarization state, any pair of pure states orthogonal to it has necessarily a common overall azimuth parameter. This facilitates the geometric representations of the orthonormal sets under study and leads to particularly interesting consequences about the spatial distribution of the respective polarization ellipses and spin vectors.

\section{BACKGROUND CONCEPTS}

Fully polarized (pure) states are commonly represented with respect to a coordinate system $X Y Z$, whose axes $X$ and $Y$ contain the corresponding polarization ellipse, while the $Z$ axis coincides with the propagation direction. Thus, in this case, as indicated above, it is enough to consider a twocomponent (2D) Jones vector [20], but obviously the same pure state can be represented in any other reference frame by means of a 3D Jones vector $[1,21]$. Partially polarized (mixed) $3 \mathrm{D}$ states require a mathematical treatment beyond the use of Jones vectors. The temporal polarization properties of such states are instead fully characterized by the polarization matrix $\mathbf{R}=\left\langle\mathcal{\varepsilon}(t) \otimes \mathcal{E}^{\dagger}(t)\right\rangle$, where $\mathcal{\varepsilon}(t) \equiv\left[\varepsilon_{x}(t), \varepsilon_{y}(t), \varepsilon_{z}(t)\right]^{\mathrm{T}}$ is the $3 \mathrm{D}$ instantaneous Jones vector representing the electric field at time $t$, while the angle brackets, symbol $\otimes$, and dagger $\dagger$ stand for the time average, Kronecker product, and conjugate transpose, respectively. Note, however, that for time intervals smaller than the polarization time [22-24] the polarization state is well defined, whereas in general it fluctuates during the measurement time; hence the name instantaneous Jones vector [2]. The matrix $\mathbf{R}$ is Hermitian with the elements $r_{i j}=\left\langle\varepsilon_{i}(t) \varepsilon_{j}^{*}(t)\right\rangle(i, j=x, y, z)$ being the second-order moments of the zero-mean electric-field components $\varepsilon_{i}(t)$. The diagonal elements of $\mathbf{R}$ are associated with the intensities of the $X Y Z$ components of the electric field, so the intensity of the whole state is given by

$$
I=\operatorname{tr} \mathbf{R}=\left\langle\left|\varepsilon_{x}(t)\right|^{2}\right\rangle+\left\langle\left|\varepsilon_{y}(t)\right|^{2}\right\rangle+\left\langle\left|\varepsilon_{z}(t)\right|^{2}\right\rangle .
$$

For an appropriate formulation of certain later expressions, it will also be useful to use the intensity-normalized form of $\mathbf{R}$, i.e., the polarization density matrix $\hat{\mathbf{R}} \equiv \mathbf{R} / I$.

While pure states are characterized by a unique Jones vector, the polarization matrix of a mixed state can be expressed in terms of a set of three orthonormal 3D Jones vectors (eigenstates) and three scalar non-negative parameters (eigenvalues) by means of its spectral decomposition [1]:

$$
\mathbf{R}=I \mathbf{U} \operatorname{diag}\left(\hat{\lambda}_{1}, \hat{\lambda}_{2}, \hat{\lambda}_{3}\right) \mathbf{U}^{\dagger}=I \sum_{i=1}^{3} \hat{\lambda}_{i}\left(\hat{\mathbf{u}}_{i} \otimes \hat{\mathbf{u}}_{i}^{\dagger}\right) .
$$

Here $\mathbf{U}$ is the unitary matrix that diagonalizes $\mathbf{R}, \hat{\mathbf{u}}_{i}$ are the unit eigenvectors of $\mathbf{R}$ (i.e., the columns of matrix $\mathbf{U}$ ), and $\hat{\lambda}_{i}$

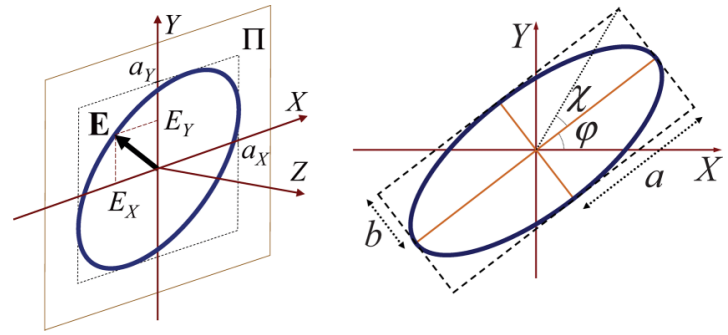

FIG. 1. In the 2D representation of polarized light, the polarization ellipse remains in a fixed plane $\Pi(X Y)$ during the measurement time, and thus there is no component of the electric field $\mathbf{E}$ along the $Z$ direction orthogonal to the plane $\Pi$. The azimuth $\varphi$ is the angle between the direction of the semimajor axis and the $X$ axis. The absolute value of the ellipticity angle $\chi$ is given by $|\chi|=\arctan (b / a)$, with $a$ and $b$ being the semimajor and semiminor axes, respectively, while the handedness is determined by the sign of $\chi$ [31].

are the eigenvalues of $\hat{\mathbf{R}}$ obeying $\hat{\lambda}_{1}+\hat{\lambda}_{2}+\hat{\lambda}_{3}=1$ and $\hat{\lambda}_{1} \geqslant$ $\hat{\lambda}_{2} \geqslant \hat{\lambda}_{3} \geqslant 0$. A detailed quantification of the polarimetric randomness (or conversely, polarimetric purity) of $\mathbf{R}$ is also achieved through its characteristic decomposition [1,25-27],

$$
\mathbf{R}=I\left[P_{1} \hat{\mathbf{R}}_{p}+\left(P_{2}-P_{1}\right) \hat{\mathbf{R}}_{m}+\left(1-P_{2}\right) \hat{\mathbf{R}}_{u-3 D}\right],
$$

$\hat{\mathbf{R}}_{p} \equiv \hat{\mathbf{u}}_{1} \otimes \hat{\mathbf{u}}_{1}^{\dagger}, \quad \hat{\mathbf{R}}_{m} \equiv \frac{1}{2}\left(\hat{\mathbf{u}}_{1} \otimes \hat{\mathbf{u}}_{1}^{\dagger}+\hat{\mathbf{u}}_{2} \otimes \hat{\mathbf{u}}_{2}^{\dagger}\right), \quad \hat{\mathbf{R}}_{u-3 D} \equiv \frac{1}{3} \mathbf{I}$,

where $\mathbf{I}$ is the $3 \times 3$ identity matrix and $P_{1}, P_{2}$ are the so-called indices of polarimetric purity (IPP) [28], defined as

$$
P_{1}=\hat{\lambda}_{1}-\hat{\lambda}_{2}, \quad P_{2}=1-3 \hat{\lambda}_{3} .
$$

While $\hat{\mathbf{R}}_{p}$ and $\hat{\mathbf{R}}_{u-3 \mathrm{D}}$ represent, respectively, a pure state and a fully random state (i.e., a 3D unpolarized state), the interpretation of $\hat{\mathbf{R}}_{m}$ requires a more detailed analysis and leads to the notion of nonregularity of polarization states $[26,29]$. A partially polarized evanescent wave is an important physical manifestation of such nonregular polarization states [30].

As with the spectral decomposition, also the characteristic decomposition is formulated in terms of the Jones eigenvectors of $\mathbf{R}$, in particular of the pair $\left(\hat{\mathbf{u}}_{1}, \hat{\mathbf{u}}_{2}\right)$ which determines univocally the third component $\hat{\mathbf{u}}_{3}$ of the complete orthonormal set (this is the reason why the numbers of free parameters involved in the spectral and characteristic decompositions coincide). Hence, owing to this intrinsic mathematical structure and physical importance of the two representations, the identification and geometric interpretation of the sets of orthonormal 3D Jones vectors is essential for a detailed understanding of the fundamental properties of partially polarized 3D light states.

Next, as necessary concepts to achieve the results of this work, we examine the intrinsic and general representations of a 3D Jones vector. There are no mathematical restrictions for an arbitrary three-component complex vector to be considered a 3D Jones vector $\varepsilon$ that represents a certain fully polarized state. Such a state is characterized by the so-called polarization ellipse, which is described by the temporal evolution of the end point of the electric-field vector of the electromagnetic wave (Fig. 1, [31]). As said in the Introduction, the 


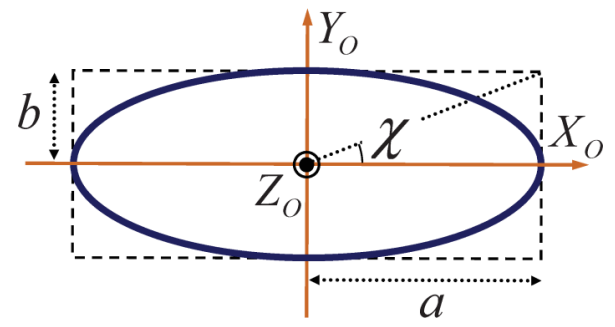

FIG. 2. The polarization ellipse represented with respect to the intrinsic reference frame $X_{O} Y_{O} Z_{O}$ of the corresponding pure polarization state $\varepsilon_{O}$ [31].

conventional $2 \mathrm{D}$ model is easily recovered by taking the $Z$ axis in a direction orthogonal to the plane $\Pi(X Y)$ containing the polarization ellipse, so that the third component of the $3 \mathrm{D}$ Jones vector vanishes.

Let $\left(\hat{\mathbf{e}}_{x}, \hat{\mathbf{e}}_{y}, \hat{\mathbf{e}}_{z}\right)$ and $\left(\hat{\mathbf{e}}_{x_{O}}, \hat{\mathbf{e}}_{y_{O}}, \hat{\mathbf{e}}_{z_{O}}\right)$ be the sets of orthonormal unit vectors characterizing the state $\varepsilon$ with respect to an arbitrary reference frame $X Y Z$ and the intrinsic reference frame $X_{O} Y_{O} Z_{O}[2,11,12]$. The plane $X_{O} Y_{O}$ contains the polarization ellipse of $\varepsilon$ such that the $X_{O}$ axis runs parallel with the semimajor axis of the ellipse (Fig. 2, [31]). The intrinsic Jones vector, i.e., the Jones vector of a given pure polarization state in the intrinsic reference frame, adopts the form

$$
\varepsilon_{O}=\sqrt{I} e^{i \gamma}(\cos \chi, i \sin \chi, 0)^{\mathrm{T}},
$$

which depends only on a global phase $\gamma$, the intensity $I$, and the ellipticity angle $-\pi / 4 \leqslant \chi \leqslant \pi / 4$, with the value of $\chi$ determining the ellipticity and the handedness of the state (positive and negative values of $\chi$ correspond, respectively, to right-handed and left-handed elliptically polarized states [2]). Due to the convention $-\pi / 4 \leqslant \chi \leqslant \pi / 4$ taken here, the absolute value of the first component of $\varepsilon_{O}$ is never smaller than that of the second component $(\cos \chi \geqslant|\sin \chi|)$, thus avoiding ambiguity for the choice of axes $X_{O}$ and $Y_{O}$. The spin angular momentum $\mathbf{n}$ of the state [2,5] lies on the $Z_{O}$ axis (normal to the plane $X_{O} Y_{O}$ containing the polarization ellipse). Since in subsequent sections we consider sets of orthonormal polarization states, we henceforth set $I=1$ and denote the unit Jones vector as $\hat{\varepsilon}$.

Once the intrinsic unit Jones vector $\hat{\varepsilon}_{O}$ has been specified, the following step is to establish the link between $\hat{\varepsilon}_{O}$ and the expression $\hat{\varepsilon}$ for the same state in an arbitrary Cartesian reference frame $X Y Z$. The transformation from $\hat{\varepsilon}_{O}$ to $\hat{\varepsilon}$ is obtained through the corresponding rotation from $X_{O} Y_{O} Z_{O}$ to $X Y Z$. Such a rotation is determined by (1) a rotation of angle $-\varphi$ ( $\varphi$ being the azimuth of the polarization ellipse, $0 \leqslant \varphi<$ $\pi$ ) about the $Z_{O}$ axis, (2) a rotation of angle $\theta$ (elevation of the new $Z$ axis, $-\pi / 2 \leqslant \theta \leqslant \pi / 2$ ) about the transformed $Y$ axis, and (3) a rotation of angle $\phi$ (azimuth of the new $X$ axis, $-\pi<\phi \leqslant \pi)$ about the transformed $Z$ axis. Thus, the unit vector $\hat{\mathbf{e}}_{z}(\theta, \phi)$ that specifies the direction of the $Z$ axis with respect to the plane $X_{O} Y_{O}$ of the intrinsic reference frame is determined by the pair of angles $(\theta, \phi)$ [31].

The conventions taken here for the angular parameters allow one to recover the most common expressions for the angular configurations and to interpret them in a natural way (slightly different conventions are used in some related works
$[2,21])$. Note that in [31] the first rotation of the transformation should be considered $-\varphi$ instead of $\varphi$, but the mathematical expressions in [31] remain valid provided the indicated convention is taken.

The orthogonal matrix corresponding to the above indicated rotation transformation from $X_{O} Y_{O} Z_{O}$ to $X Y Z$ is [31]

$$
\begin{aligned}
& \mathbf{Q}(\phi, \theta, \varphi) \\
& \quad=\left(\begin{array}{ccc}
c_{\theta} c_{\phi} c_{\varphi}+s_{\phi} s_{\varphi} & -c_{\theta} c_{\phi} s_{\varphi}+s_{\phi} c_{\varphi} & c_{\phi} s_{\theta} \\
-c_{\theta} s_{\phi} c_{\varphi}+c_{\phi} s_{\varphi} & c_{\theta} s_{\phi} s_{\varphi}+c_{\phi} c_{\varphi} & -s_{\phi} s_{\theta} \\
-s_{\theta} c_{\varphi} & s_{\theta} s_{\varphi} & c_{\theta}
\end{array}\right),
\end{aligned}
$$

where the trigonometric functions are represented by the abbreviated notations $s_{x} \equiv \sin x, c_{x} \equiv \cos x$. The resulting general expression for $\hat{\varepsilon}$ with respect to an arbitrary coordinate system $X Y Z$ thus reads

$$
\begin{aligned}
& \hat{\varepsilon}=\mathbf{Q} \hat{\varepsilon}_{O} \\
&=e^{i \gamma}\left(\begin{array}{c}
c_{\chi}\left(s_{\phi} s_{\varphi}+c_{\theta} c_{\phi} c_{\varphi}\right)+i s_{\chi}\left(s_{\phi} c_{\varphi}-c_{\theta} c_{\phi} s_{\varphi}\right) \\
c_{\chi}\left(c_{\phi} s_{\varphi}-c_{\theta} s_{\phi} c_{\varphi}\right)+i s_{\chi}\left(c_{\phi} c_{\varphi}+c_{\theta} s_{\phi} s_{\varphi}\right) \\
-c_{\chi} s_{\theta} c_{\varphi}+i s_{\chi} s_{\theta} s_{\varphi}
\end{array}\right),
\end{aligned}
$$

with $\hat{\varepsilon}_{O}$ being the unit Jones vector in the intrinsic reference frame $X_{O} Y_{O} Z_{O}$, as before.

The procedure for the determination of the angular parameters $(\phi, \theta, \varphi)$ of a given 3D Jones vector $\hat{\varepsilon}$ is described in Appendix A.

\section{SETS OF ORTHONORMAL 3D JONES VECTORS}

Equation (7) represents a general unit Jones vector in terms of the angles $(\theta, \phi)$ of the vector $\hat{\mathbf{e}}_{z}(\theta, \phi)$ that determines the $Z$ axis, together with the associated azimuth $\varphi$ and ellipticity angle $\chi$. As seen in Sec. II, this pure state, when represented with respect to its corresponding intrinsic reference frame $X_{1} Y_{1} Z_{1}$ (i.e., $\varphi_{1}=\phi_{1}=\theta_{1}=0$ ), reads

$$
\hat{\eta}_{1}=e^{i \gamma_{1}}\left(\cos \chi_{1}, i \sin \chi_{1}, 0\right)^{\mathrm{T}},
$$

where the symbol $\hat{\eta}$ is now used instead of $\hat{\varepsilon}$ to refer to the three orthonormal Jones vectors that constitute the canonical set [31]. Furthermore, it is well known that the Jones vector $\hat{\boldsymbol{\eta}}_{2}$ whose polarization ellipse lies in the same plane $X_{1} Y_{1}$ (viz., $\theta_{2}=\phi_{2}=0$ ), with azimuth $\varphi_{2}=\pi / 2$ and with ellipticity angle $\chi_{2}=-\chi_{1}$, is orthogonal to $\hat{\eta}_{1}$ and has the form

$$
\hat{\boldsymbol{\eta}}_{2}=e^{i \gamma_{2}}\left(i \sin \chi_{1}, \cos \chi_{1}, 0\right)^{\mathrm{T}} \text {. }
$$

The canonical set is completed by adding to the pair $\left(\hat{\eta}_{1}, \hat{\eta}_{2}\right)$ a linearly polarized state $\hat{\eta}_{3}$ along the $Z_{1}$ direction, whose $3 \mathrm{D}$ Jones vector is

$$
\hat{\eta}_{3}=e^{i \gamma_{3}}(0,0,1)^{\mathrm{T}} \text {. }
$$

It should be stressed that, as occurs in the common 2D representations, the concept of orthogonality of states does not necessarily imply orthogonality between the planes containing the respective polarization ellipses.

Given a polarization matrix $\mathbf{R}$, it is always possible to determine the intrinsic reference frame $X_{1} Y_{1} Z_{1}$ corresponding to the first eigenvector $\hat{\eta}_{1}$, and then use the canonical set $\left(\hat{\eta}_{1}, \hat{\eta}_{2}, \hat{\eta}_{3}\right)$ defined with respect to it [31] (Fig. 3). Note that while $\hat{\eta}_{1}$ is associated with the intrinsic representation of the 

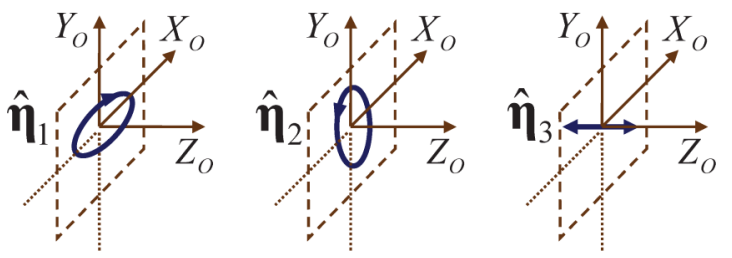

FIG. 3. Canonical set of orthonormal polarization states, represented with respect to the intrinsic reference frame $X_{1} Y_{1} Z_{1}$ of $\hat{\eta}_{1}$ $\left(\theta_{1}=\phi_{1}=\varphi_{1}=0\right)$. State $\hat{\boldsymbol{\eta}}_{2}$ corresponds to either of the configurations $\left(\theta_{2}=\phi_{2}=0, \varphi_{2}=\pi / 2, \chi_{2}=-\chi_{1}\right),\left(\theta_{2}=0, \phi_{2}=\pi / 2\right.$, $\left.\varphi_{2}=0, \chi_{2}=-\chi_{1}\right)$, while $\hat{\eta}_{3}$ corresponds to the configuration $\left(\theta_{3}=\right.$ $\left.\pm \pi / 2, \varphi_{3}=0, \chi_{3}=0\right)$ with $\phi_{3}$ arbitrary [31].

first spectral component $\hat{\mathbf{u}}_{1}$ [see Eq. (2)], $\hat{\boldsymbol{\eta}}_{2}$ and $\hat{\boldsymbol{\eta}}_{3}$ do not necessarily correspond to the spectral components $\hat{\mathbf{u}}_{2}$ and $\hat{\mathbf{u}}_{3}$.

Once the canonical set $\left(\hat{\boldsymbol{\eta}}_{1}, \hat{\boldsymbol{\eta}}_{2}, \hat{\boldsymbol{\eta}}_{3}\right)$ has been defined, in this section we address the problem of identifying arbitrary sets of mutually orthonormal polarization states. To this end, we take $\hat{\eta}_{1}$ as the first basis vector such that it fixes the coordinate system $X_{1} Y_{1} Z_{1}$ used in the analysis below.

Any generic Jones vector $\hat{v}_{i}$, orthonormal to $\hat{\eta}_{1}$, can be expressed as a linear combination of the two remaining canonical vectors $\left(\hat{\boldsymbol{\eta}}_{2}, \hat{\boldsymbol{\eta}}_{3}\right)$ as

$$
\begin{aligned}
\hat{\mathbf{v}}_{i} & =\cos \mu e^{i \alpha_{i}} \hat{\boldsymbol{\eta}}_{2}+\sin \mu e^{i \beta_{i}} \hat{\boldsymbol{\eta}}_{3} \\
& =\left(\begin{array}{c}
c_{\mu} s_{\chi_{1}}\left(-s_{\alpha_{i}}+i c_{\alpha_{i}}\right) \\
c_{\mu} c_{\chi_{1}}\left(c_{\alpha_{i}}+i s_{\alpha_{i}}\right) \\
s_{\mu}\left(c_{\beta_{i}}+i s_{\beta_{i}}\right)
\end{array}\right),
\end{aligned}
$$

with $0 \leqslant \mu \leqslant \pi / 2$ and $0 \leqslant \alpha_{i}, \beta_{i} \leqslant \pi$. In the last expression we used the simplified notation $\alpha_{i} \equiv \alpha_{i}+\gamma_{2}, \beta_{i} \equiv \beta_{i}+\gamma_{3}$. On the other hand, according to Eq. (7), the expression of $\hat{\boldsymbol{v}}_{i}$ in terms of its corresponding angles $\phi_{i}, \theta_{i}, \varphi_{i}, \chi_{i}$ and an arbitrary global phase $\delta_{i}$ reads

$$
\hat{\boldsymbol{v}}_{i}=e^{i \delta_{i}}\left(\begin{array}{c}
c_{\chi_{i}}\left(s_{\phi_{i}} s_{\varphi_{i}}+c_{\theta_{i}} c_{\phi_{i}} c_{\varphi_{i}}\right)+i s_{\chi_{i}}\left(s_{\phi_{i}} c_{\varphi_{i}}-c_{\theta_{i}} c_{\phi_{i}} s_{\varphi_{i}}\right) \\
c_{\chi_{i}}\left(c_{\phi_{i}} s_{\varphi_{i}}-c_{\theta_{i}} s_{\phi_{i}} c_{\varphi_{i}}\right)+i s_{\chi_{i}}\left(c_{\phi_{i}} c_{\varphi_{i}}+c_{\theta_{i}} s_{\phi_{i}} s_{\varphi_{i}}\right) \\
-c_{\chi_{i}} s_{\theta_{i}} c_{\varphi_{i}}+i s_{\chi_{i}} s_{\theta_{i}} s_{\varphi_{i}}
\end{array}\right) .
$$

Hence, by combining Eqs. (11) and (12) (and omitting the global phase factor $e^{i \delta_{i}}$ ), we obtain the following set of coupled trigonometric equations:

$$
\begin{aligned}
-c_{\mu} s_{\alpha_{i}} s_{\chi_{1}} & =c_{\chi_{i}}\left(s_{\phi_{i}} s_{\varphi_{i}}+c_{\theta_{i}} c_{\phi_{i}} c_{\varphi_{i}}\right), \\
c_{\mu} c_{\alpha_{i}} s_{\chi_{1}} & =s_{\chi_{i}}\left(s_{\phi_{i}} c_{\varphi_{i}}-c_{\theta_{i}} c_{\phi_{i}} s_{\varphi_{i}}\right), \\
c_{\mu} c_{\alpha_{i}} c_{\chi_{1}} & =c_{\chi_{i}}\left(c_{\phi_{i}} s_{\varphi_{i}}-c_{\theta_{i}} s_{\phi_{i}} c_{\varphi_{i}}\right), \\
c_{\mu} s_{\alpha_{i}} c_{\chi_{1}} & =s_{\chi_{i}}\left(c_{\phi_{i}} c_{\varphi_{i}}+c_{\theta_{i}} s_{\phi_{i}} s_{\varphi_{i}}\right), \\
c_{\beta_{i}} s_{\mu} & =-c_{\chi_{i}} s_{\theta_{i}} c_{\varphi_{i}}, \\
s_{\beta_{i}} s_{\mu} & =s_{\chi_{i}} s_{\theta_{i}} s_{\varphi_{i}} .
\end{aligned}
$$

The aim of the subsequent analysis is to determine the ellipticity angle $\chi_{i}$ and the azimuth $\varphi_{i}$ of the polarization ellipse that corresponds to any set of parameters $\left(\chi_{1}, \phi_{i}, \theta_{i}\right)$, where $\chi_{1}$ is the ellipticity angle of the reference polarization state $\hat{\eta}_{1}\left(\varphi_{1}=0\right)$, and where the pair of angles $\left(\phi_{i}, \theta_{i}\right)$ determines, with respect to the reference frame $X_{1} Y_{1} Z_{1}$, the orientation of the $Z_{i}$ axis orthogonal to the plane $\Pi_{i}$ containing the polarization ellipse of the state $\hat{v}_{i}$.

Prior to studying the features of the families of solutions of Eq. (13), it is important to note that the form of the equations corresponds to the choice made for the order of the 3D Jones vectors constituting the canonical set, where, except for the case where all $\left(\hat{\boldsymbol{\eta}}_{1}, \hat{\boldsymbol{\eta}}_{2}, \hat{\boldsymbol{\eta}}_{3}\right)$ are linearly polarized, $\hat{\boldsymbol{\eta}}_{1}$ is not linearly polarized. Such choice does not impose limits or restrictions to the scope of validity of Eq. (13), but hereafter it should be taken into account for their interpretation and graphical representation.

Let us also observe that when the absolute value of the elevation angle is maximal, i.e., $\left|\theta_{i}\right|=\pi / 2$, the equations in system (13) are only compatible with $\chi_{i}=0$ and $\varphi_{i}=0$ ( $\mu=\pi / 2$ ), corresponding to linearly polarized states. Moreover, values $\mu=0, \pi / 2$ correspond to the canonical set (i.e., $\left.\theta_{i}=0, \pm \pi / 2\right)$ and therefore they can be excluded from the following analysis.

Due to the trigonometric nature of Eq. (13), it is appropriate to consider first the particular cases (1) $\phi_{i}=0, \pi$, and (2) $\phi_{i}= \pm \pi / 2$.

(1) When $\phi_{i}=0, \pi$, Eq. (13) adopts the form

$$
\begin{gathered}
-c_{\mu} s_{\alpha_{i}} s_{\chi_{1}}= \pm c_{\chi_{i}} c_{\theta_{i}} c_{\varphi_{i}}, \quad c_{\mu} c_{\alpha_{i}} s_{\chi_{1}}=\mp s_{\chi_{i}} c_{\theta_{i}} s_{\varphi_{i}}, \\
c_{\mu} c_{\alpha_{i}} c_{\chi_{1}}= \pm c_{\chi_{i}} s_{\varphi_{i}}, \quad c_{\mu} s_{\alpha_{i}} c_{\chi_{1}}= \pm s_{\chi_{i}} c_{\varphi_{i}}, \\
c_{\beta_{i}} s_{\mu}=-c_{\chi_{i}} s_{\theta_{i}} c_{\varphi_{i}}, s_{\beta_{i}} s_{\mu}=s_{\chi_{i}} s_{\theta_{i}} s_{\varphi_{i}} .
\end{gathered}
$$

Provided that $0<\mu<\pi / 2, \quad \chi_{1} \neq 0$, and $\theta_{i} \neq 0, \pm \pi / 2$, Eq. (14) leads to the following values for $\chi_{i}$ and $\varphi_{i}$ in terms of $\theta_{i}$ :

$$
\begin{aligned}
& \text { (a) } c_{\theta_{i}}<\left|t_{\chi_{1}}\right| \Rightarrow \varphi_{i}=0, t_{\chi_{i}}=-c_{\theta_{i}} / t_{\chi_{1}}, \\
& \text { (b) } c_{\theta_{i}}=\left|t_{\chi_{1}}\right| \Rightarrow \chi_{i}= \pm \pi / 4 \text { ( } \varphi_{i} \text { undet.), } \\
& \text { (c) } c_{\theta_{i}}>\left|t_{\chi_{1}}\right| \Rightarrow \varphi_{i}=\pi / 2, t_{\chi_{i}}=-t_{\chi_{1}} / c_{\theta_{i}},
\end{aligned}
$$

where $t_{x} \equiv \tan x$ and $t_{x}^{-1} \equiv \cot x$. The solution (b) does not correspond to a family of states, as occurs with (a) and (c), but corresponds to two particular values of $\theta_{i}$ for which $\hat{v}_{i}$ is a circularly polarized state ( $\varphi_{i}$ undetermined). The particular case (b) is analyzed within the example (a) of Sec. V.

Hence, except for the particular case (b) in Eq. (15), in general the family of solutions for $\phi_{i}=0, \pi$ implies that $\varphi_{i}=0, \pi / 2$. The family of states $\hat{\boldsymbol{v}}_{i}$ corresponding to $\phi_{i}=0$ is analyzed in Sec. V, where the ellipticity angle $\chi_{i}$ of $\hat{v}_{i}$ runs through all its possible values, including the particular case (b) of circular polarization $\left(\left|\chi_{i}\right|=\pi / 4\right)$.

(2) When $\phi_{i}= \pm \pi / 2$, Eq. (13) becomes

$$
\begin{gathered}
-c_{\mu} s_{\alpha_{i}} s_{\chi_{1}}= \pm c_{\chi_{i}} s_{\varphi_{i}}, \quad c_{\mu} c_{\alpha_{i}} s_{\chi_{1}}= \pm s_{\chi_{i}} c_{\varphi_{i}}, \\
c_{\mu} c_{\alpha_{i}} c_{\chi_{1}}=\mp c_{\chi_{i}} c_{\theta_{i}} c_{\varphi_{i}}, \quad c_{\mu} s_{\alpha_{i}} c_{\chi_{1}}= \pm s_{\chi_{i}} c_{\theta_{i}} s_{\varphi_{i}}, \\
c_{\beta_{i}} s_{\mu}=-c_{\chi_{i}} s_{\theta_{i}} c_{\varphi_{i}}, \quad s_{\beta_{i}} s_{\mu}=s_{\chi_{i}} s_{\theta_{i}} s_{\varphi_{i}} .
\end{gathered}
$$

Presuming again that $0<\mu<\pi / 2, \quad \chi_{1} \neq 0$, and $\theta_{i} \neq$ $0, \pm \pi / 2$, Eq. (16) yields the following values for $\chi_{i}$ and $\varphi_{i}$ in terms of $\theta_{i}$ :

$$
\begin{aligned}
\text { (a) } c_{\theta_{i}} & = \pm 1 / t_{\chi_{1}}\left(\left|t_{\chi_{1}}\right| \leqslant 1\right) \\
\Rightarrow \theta_{i} & =0, \quad \chi_{i}=-\chi_{1}=\mp \pi / 4\left(\varphi_{i} \text { undet. }\right),
\end{aligned}
$$




$$
\begin{aligned}
\text { (b) } t_{\chi_{i}} & =-1 / t_{\chi_{1}} c_{\theta_{i}}\left(\left|t_{\chi_{1}}\right| \leqslant 1, \quad c_{\theta_{i}} \leqslant 1\right) \\
\Rightarrow \theta_{i} & =0, \quad \chi_{i}=-\chi_{1}=\mp \pi / 4\left(\varphi_{i} \text { undet. }\right), \\
\text { (c) } t_{\chi_{i}} & =-t_{\chi_{1}} c_{\theta_{i}}, \quad \varphi_{i}=0 .
\end{aligned}
$$

The mere definition of ellipticity angles $(|\chi| \leqslant \pi / 4)$ implies $\left|\tan \chi_{1}\right| \leqslant 1$ and $\left|\tan \chi_{i}\right| \leqslant 1$, whereby the solutions (a) and (b) of Eq. (17) are only compatible with the particular case $\chi_{1}= \pm \pi / 4$, which in turn entails $\theta_{i}=0$ (against the hypothesis). Note that such excluded solutions $(a, b)$ would correspond to the specific canonical set where $\hat{\eta}_{1}$ is circularly polarized, $\hat{\eta}_{2}$ is circularly polarized with opposite handedness $\left(\chi_{2}=-\chi_{1}=\mp \pi / 4\right)$, and $\hat{\eta}_{3}$ is linearly polarized.

Therefore, the family of solutions for $\phi_{i}= \pm \pi / 2$ dictates that $\varphi_{i}=0$, while $\left|\chi_{i}\right|$ varies continuously from its maximum $\left|\chi_{i}\right|=\left|\chi_{1}\right|\left(\theta_{i}=0\right)$ down to its minimum $\chi_{i}=0$ $\left(\left|\theta_{i}\right|=\pi / 2\right)$. Moreover, from the inspection of the compatibility of Eq. (13), it can be shown that sets of orthonormal linearly polarized states always correspond to the family $\phi_{i}=$ $\pm \pi / 2$. The family of states $\hat{v}_{i}$ with $\phi_{i}=\pi / 2$ is analyzed in detail in Sec. V.

In general, provided that $\mu<\pi / 2$ and $\alpha_{i} \neq 0, \pi / 2, \pi$, Eq. (13) can be transformed into

$$
\begin{aligned}
t_{\chi_{i}}\left(s_{\phi_{i}} c_{\varphi_{i}}-c_{\theta_{i}} c_{\phi_{i}} s_{\varphi_{i}}\right) & =t_{\chi_{1}}\left(c_{\phi_{i}} s_{\varphi_{i}}-c_{\theta_{i}} s_{\phi_{i}} c_{\varphi_{i}}\right), \\
t_{\chi_{i}}^{-1}\left(s_{\phi_{i}} s_{\varphi_{i}}+c_{\theta_{i}} c_{\phi_{i}} c_{\varphi_{i}}\right) & =-t_{\chi_{1}}\left(c_{\phi_{i}} c_{\varphi_{i}}+c_{\theta_{i}} s_{\phi_{i}} s_{\varphi_{i}}\right),
\end{aligned}
$$

which leads to

$$
\begin{aligned}
& \left(s_{\phi_{i}} c_{\varphi_{i}}-c_{\theta_{i}} c_{\phi_{i}} s_{\varphi_{i}}\right)\left(s_{\phi_{i}} s_{\varphi_{i}}+c_{\theta_{i}} c_{\phi_{i}} c_{\varphi_{i}}\right) \\
& \quad=-t_{\chi_{1}}^{2}\left(c_{\phi_{i}} s_{\varphi_{i}}-c_{\theta_{i}} s_{\phi_{i}} c_{\varphi_{i}}\right)\left(c_{\phi_{i}} c_{\varphi_{i}}+c_{\theta_{i}} s_{\phi_{i}} s_{\varphi_{i}}\right) .
\end{aligned}
$$

Both sides of this equation can be divided by $c_{\phi_{i}}^{2} c_{\varphi_{i}}^{2}$ (remember that the cases $\phi_{i}= \pm \pi / 2$ and $\varphi_{i}=\pi / 2$ have been analyzed above separately), and transformed into a second-order equation for $t_{\varphi_{i}}$,

$$
\begin{gathered}
a t_{\varphi_{i}}^{2}+b t_{\varphi_{i}}-a=0, \\
{\left[a \equiv\left(t_{\chi_{1}}^{2}-1\right) c_{\theta_{i}} t_{\phi_{i}}, \quad b \equiv t_{\chi_{1}}^{2}\left(1-c_{\theta_{i}}^{2} t_{\phi_{i}}^{2}\right)+t_{\phi_{i}}^{2}-c_{\theta_{i}}^{2}\right],}
\end{gathered}
$$

with the solution pair (determining univocally the respective solutions for $\varphi_{i}$ because of the constraint $0 \leqslant \varphi_{i}<\pi$ )

$$
t_{\varphi_{i}}=\frac{-b \pm \sqrt{b^{2}+4 a^{2}}}{2 a} .
$$

By analyzing Eqs. (20) and (13), it turns out that $a=0$ corresponds either to (1) $\left|\chi_{1}\right|=\pi / 4$ ( $\hat{\eta}_{1}$ circularly polarized, should be analyzed separately); (2) $\left|\theta_{i}\right|=\pi / 2$ and $t_{\chi_{i}}^{2}=-1$ (unphysical), or (3) $\phi_{i}=0, \pi$ (already studied). Thus, for the sake of consistency of Eq. (21), these cases are excluded from the analysis performed below. The corresponding values for $\chi_{i}$ are obtained by dividing both sides of the first relation in Eq. (18) by $c_{\phi_{i}} c_{\varphi_{i}}$, which leads to

$$
t_{\chi_{i}}=t_{\chi_{1}} \frac{t_{\varphi_{i}}-c_{\theta_{i}} t_{\phi_{i}}}{t_{\phi_{i}}-c_{\theta_{i}} t_{\varphi_{i}}},
$$

with the above-indicated conditions of validity $(\mu<\pi / 2$; $\left.\alpha_{i} \neq 0, \pi / 2, \pi ; \phi_{i} \neq 0, \pm \pi / 2, \pi ; \varphi_{i}=\pi / 2\right)$ ensuring the consistency of Eq. (22).

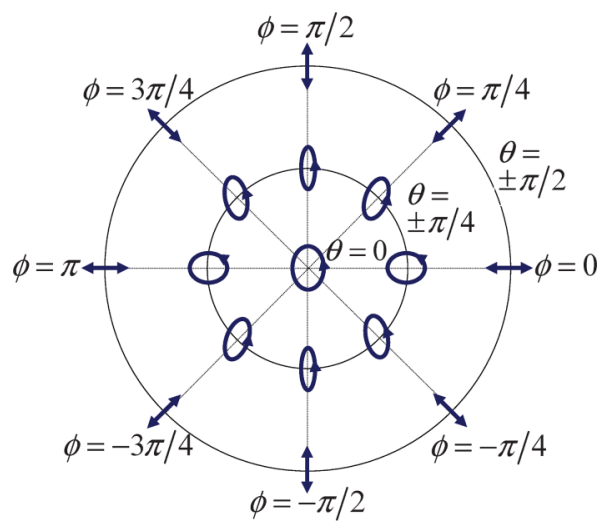

FIG. 4. Representation of the polarization ellipses of states $\hat{v}(\chi, \varphi, \phi, \theta)$ that are orthogonal to $\hat{\eta}_{1}$ for different values of $\phi$ and $\theta$. States in a given circle have equal elevation angle $\theta$, while states along straight lines from the origin have equal overall azimuth $\phi$. Each ellipse lies in a respective plane whose normal is given by its direction vector $\hat{\mathbf{e}}_{z}(\theta, \phi)$, so that such planes are tangent to a common sphere and, from a topological point of view, cover all points of this sphere.

The parameters $\varphi_{2}$ and $\chi_{2}$ can now be calculated from Eqs. (21) and (22) as functions of $\chi_{1}, \phi_{2}, \theta_{2}$. Moreover, since $\hat{\eta}_{1}, \hat{v}_{2}, \hat{v}_{3}$ constitute the columns of a unitary matrix, the components of $\hat{v}_{3}$ can always be determined through the procedure described in [31]. Thus, once $\chi_{1}, \phi_{2}, \theta_{2}$ have been specified and the angles $\varphi_{2}$ and $\chi_{2}$ have been calculated, the corresponding parameters $\phi_{3}, \theta_{3}, \varphi_{3}, \chi_{3}$ are ascertained by means of the method presented in Appendix A. The respective sets of families of mutually orthogonal states $\left(\hat{\boldsymbol{\eta}}_{1}, \hat{\mathbf{v}}_{2}, \hat{\mathbf{v}}_{3}\right)$ are thereby thoroughly identified and interpreted in geometrical terms.

Figure 4 illustrates polarization ellipses of states $\hat{v}(\chi, \varphi, \phi, \theta)$ that are orthogonal to $\hat{\eta}_{1}$, where the concentric circles represent states with an equal elevation angle $\theta$, while the straight lines from the origin correspond to states with a fixed overall azimuth $\phi$. In all scenarios the ellipticity angle $\chi$ reaches zero at $|\theta|=\pi / 2$, corresponding to linear polarization. For states with $\phi=0, \pi$ [horizontal line in Fig. 4, Eq. (15)], two branches with respective azimuths $\varphi=\pi / 2$ and $\varphi=0$ are separated by an intermediate circular state, such that the ellipticity angle first increases with $|\theta|$ up to $|\chi|=\pi / 4$ before decreasing down to $\chi=0$ at $|\theta|=\pi / 2$. For states with $|\phi|=\pi / 2$ [vertical line in Fig. 4, Eq. (17)], we have $\varphi=0$ and the ellipticity angle reduces monotonically from its maximum at $\theta=0$ toward $\chi=0$ when $|\theta|=\pi / 2$. For states corresponding to the oblique lines in Fig. 4, both $\varphi$ and $\chi$ vary continuously as a function of $|\theta|$. The direction of the spin angular momentum vector of $\hat{v}$, which is normal to the plane containing the corresponding polarization ellipse, is determined by the pair $\phi, \theta$.

The above analysis shows that, given an arbitrary pure polarization state $\hat{\eta}_{1}$ (represented with respect to its own intrinsic reference frame) and an arbitrary plane $\Pi_{2}$, this plane contains the polarization ellipse of a pure state $\hat{\boldsymbol{v}}_{2}$ orthogonal to $\hat{\eta}_{1}$. The values of the azimuth $\varphi_{2}$ and ellipticity angle $\chi_{2}$ of $\hat{v}_{2}$ depend on $\chi_{1}$ and on the angles $\phi_{2}, \theta_{2}$ determining the plane $\Pi_{2}$. In addition, since any pair of columns of a unitary 
matrix determines the remaining column, once $\hat{\eta}_{1}$ and $\Pi_{2}$ are specified, the plane $\Pi_{3}$ containing the polarization ellipse of the third orthonormal state $\hat{v}_{3}$ together with its azimuth $\varphi_{3}$ and ellipticity angle $\chi_{3}$ are univocally determined.

As demonstrated in Appendix B, the overall azimuths $\phi_{2}$ and $\phi_{3}$ of the components $\hat{\boldsymbol{v}}_{2}$ and $\hat{\boldsymbol{v}}_{3}$ of the orthonormal set of 3D Jones vectors $\left(\hat{\boldsymbol{\eta}}_{1}, \hat{\boldsymbol{v}}_{2}, \hat{\boldsymbol{v}}_{3}\right)$ always coincide $\left(\phi_{3}=\phi_{2}\right)$.

\section{SETS OF ORTHOGONAL LINEAR STATES}

Sets of orthogonal linearly polarized states constitute a particularly interesting case that is briefly analyzed in this section. Given a linearly polarized reference state $\hat{\boldsymbol{\eta}}_{1}=$ $e^{i \gamma_{1}}(1,0,0)^{\mathrm{T}}$, an orthonormal set of linearly polarized states $\left(\chi_{1}=\chi_{2}=\chi_{3}=0\right)$ has necessarily the form $\left(\hat{\boldsymbol{\eta}}_{1}, \hat{\boldsymbol{v}}_{2}, \hat{\boldsymbol{v}}_{3}\right)$, where

$$
\begin{aligned}
& \hat{\mathbf{v}}_{2}=e^{i \gamma_{2}}\left(0, \cos \theta_{2}, \sin \theta_{2}\right)^{\mathrm{T}}, \\
& \hat{\mathbf{v}}_{3}=e^{i \gamma_{3}}\left(0,-\sin \theta_{2}, \cos \theta_{2}\right)^{\mathrm{T}} .
\end{aligned}
$$

By comparing these expressions with those of Eq. (12) (with $i=2,3$ ), it turns out that they are only compatible if the characteristic angles of $\hat{v}_{2}$ and $\hat{v}_{3}$ satisfy

$$
\begin{gathered}
\phi_{2}=\phi_{3}= \pm \pi / 2, \varphi_{2}=\varphi_{3}=0, \\
\theta_{3}=\theta_{2} \pm \pi / 2\left(0 \leqslant \theta_{i} \leqslant \pi / 2, i=2,3\right) .
\end{gathered}
$$

Note that, due to the criterion used to define the generic first component $\hat{\boldsymbol{\eta}}_{1}$ of the canonical set $\left(\hat{\boldsymbol{\eta}}_{1}, \hat{\boldsymbol{\eta}}_{2}, \hat{\boldsymbol{\eta}}_{3}\right)$, when $\hat{\boldsymbol{\eta}}_{1}$ is linearly polarized $\left(\chi_{1}=0\right)$ Eq. (12) implies that $\chi_{2}=\chi_{3}=$ 0 . Nevertheless, the representation of any set composed of a linearly polarized state and two states with respective nonzero ellipticity angles is always achievable by taking $\hat{\boldsymbol{v}}_{2}$ or $\hat{\boldsymbol{v}}_{3}$ as the linearly polarized one.

Concerning orthonormal sets where all the components are circularly polarized $\left(\left|\chi_{1}\right|=\left|\chi_{2}\right|=\left|\chi_{3}\right|=\pi / 4\right)$, it follows from Eq. (B2), in Appendix B, that this possibility is not mathematically achievable and therefore it is not physically realizable. Orthonormal sets where two components are circularly polarized, necessarily correspond to canonical sets with $\chi_{1}= \pm \pi / 4, \chi_{2}=\mp \pi / 4$, and $\chi_{3}=0$.

\section{FAMILIES OF ORTHONORMAL STATES CORRESPONDING TO $\phi_{2}=0$ AND $\phi_{2}=\pi / 2$}

To elucidate further the nature and physical interpretation of the sets of orthonormal 3D polarization states, in this section we consider the cases $\phi_{2}=0$ and $\phi_{2}=\pi / 2$ in more detail. As before, the first canonical state $\hat{\boldsymbol{\eta}}_{1}$ fixes the reference frame for the associated states $\hat{\boldsymbol{v}}_{2}$ and $\hat{\boldsymbol{v}}_{3}$.

(a) Family of states orthonormal to $\hat{\eta}_{1}$ and with $\phi_{2}=0$.

In this case, according to Eq. (12), $\hat{v}_{2}$ adopts the form

$$
\hat{\mathbf{v}}_{2}=e^{i \delta_{2}}\left(\begin{array}{c}
c_{\chi_{2}} c_{\theta_{2}} c_{\varphi_{2}}-i s_{\chi_{2}} c_{\theta_{2}} s_{\varphi_{2}} \\
c_{\chi_{2}} s_{\varphi_{2}}+i s_{\chi_{2}} c_{\varphi_{2}} \\
-c_{\chi_{2}} s_{\theta_{2}} c_{\varphi_{2}}+i s_{\chi_{2}} s_{\theta_{2}} s_{\varphi_{2}}
\end{array}\right) \text {. }
$$

Equations (15) further imply that the azimuth of $\hat{\boldsymbol{v}}_{i}$ takes the achievable values $\varphi_{i}=\pi / 2,0(i=2,3)$, with $\varphi_{i}=\pi / 2$ for elevation angles satisfying $\cos \theta_{i}>\left|\tan \chi_{1}\right|$, and $\varphi_{i}=0$ when $\cos \theta_{i}<\left|\tan \chi_{1}\right|$. The equality $\cos \theta_{i}=\left|\tan \chi_{1}\right|$ [case (b) in Eq. (15)] corresponds to a circularly polarized state $\left(\left|\chi_{i}\right|=\pi / 4, \varphi_{i}\right.$ undetermined) that connects states $\hat{v}_{i}$ with $\varphi_{i}=\pi / 2$ and those with $\varphi_{i}=0$. Hence

$$
\begin{aligned}
& \hat{\mathbf{v}}_{2}=e^{i \delta_{2}}\left(\begin{array}{c}
-i s_{\chi_{2}} c_{\theta_{2}} \\
c_{\chi_{2}} \\
i s_{\chi_{2}} s_{\theta_{2}}
\end{array}\right)\left(c_{\theta_{2}} \geqslant\left|t_{\chi_{1}}\right|, \varphi_{2}=\pi / 2\right), \\
& \hat{\mathbf{v}}_{2}=e^{i \delta_{2}}\left(\begin{array}{c}
-i c_{\chi_{2}} c_{\theta_{2}} \\
s_{\chi_{2}} \\
i c_{\chi_{2}} s_{\theta_{2}}
\end{array}\right)\left(c_{\theta_{2}} \leqslant\left|t_{\chi_{1}}\right|, \varphi_{2}=0\right) .
\end{aligned}
$$

The geometric features shown in Fig. 4 for states $\hat{v}_{i}$ orthogonal to $\hat{\eta}_{1}$ suggest that $\hat{v}_{2}$ and $\hat{v}_{3}$ have equal overall azimuths $\phi_{3}=\phi_{2}=0$ (see Appendix B). From this hypothesis, together with the use of Eq. (9) of [31] to calculate $\hat{v}_{3}$ in terms of $\left(\mu, \alpha_{3}, \beta_{3}\right)$, the orthogonality of $\hat{\boldsymbol{\eta}}_{1}, \hat{\boldsymbol{v}}_{2}$, and $\hat{\boldsymbol{v}}_{3}$ implies that the set of orthonormal 3D Jones vectors corresponding to $\phi_{2}=0$ can be expressed as a function of $\chi_{1}$ and the elevation angle $\theta_{2}$ chosen for $\hat{\boldsymbol{v}}_{2}$ as

$$
\begin{aligned}
\hat{\mathbf{\eta}}_{1} & =e^{i \delta_{1}}\left(\begin{array}{c}
c_{\chi_{1}} \\
i s_{\chi_{1}} \\
0
\end{array}\right), \\
c_{\theta_{2}} & \geqslant\left|t_{\chi_{1}}\right| \Rightarrow \varphi_{2}=\pi / 2 \\
\Rightarrow \hat{\mathbf{v}}_{2} & =e^{i \delta_{2}}\left(\begin{array}{c}
-i s_{\chi_{2}} c_{\theta_{2}} \\
c_{\chi_{2}} \\
i s_{\chi_{2}} s_{\theta_{2}}
\end{array}\right)=\frac{e^{i \delta_{2}}}{\sqrt{1+s_{\chi_{1}}^{2} t_{\theta_{2}}^{2}}}\left(\begin{array}{c}
i s_{\chi_{1}} \\
c_{\chi_{1}} \\
-i s_{\chi_{1}} t_{\theta_{2}}
\end{array}\right), \\
c_{\theta_{2}} \leqslant\left|t_{\chi_{1}}\right| \Rightarrow \varphi_{2}=0 & \hat{\boldsymbol{v}}_{2}=e^{i \delta_{2}}\left(\begin{array}{c}
-i c_{\chi_{2}} c_{\theta_{2}} \\
s_{\chi_{2}} \\
i c_{\chi_{2}} s_{\theta_{2}}
\end{array}\right)=\frac{e^{i \delta_{2}}}{\sqrt{1+s_{\chi_{1}}^{2} t_{\theta_{2}}^{2}}}\left(\begin{array}{c}
i s_{\chi_{1}} \\
c_{\chi_{1}} \\
-i s_{\chi_{1}} t_{\theta_{2}}
\end{array}\right),
\end{aligned}
$$

which correspond to the particular values

$$
\begin{aligned}
\phi_{3} & =\phi_{2}=0, \quad c_{\mu}=1 / \sqrt{1+s_{\chi_{1}}^{2} t_{\theta_{2}}^{2}} \\
c_{\theta_{i}} & >\left|t_{\chi_{1}}\right| \Rightarrow \varphi_{i}=\pi / 2, \\
c_{\theta_{i}} & =\left|t_{\chi_{1}}\right| \Rightarrow\left|\chi_{i}\right|=\pi / 4, \varphi_{i} \text { undetermined, } \\
c_{\theta_{i}} & <\left|t_{\chi_{1}}\right| \Rightarrow \varphi_{i}=0, \\
\chi_{i} & =\arctan \left(-\tan \chi_{1} / \cos \theta_{i}\right) .
\end{aligned}
$$

Since the pair $\left(\hat{\boldsymbol{\eta}}_{1}, \hat{\boldsymbol{v}}_{2}\right)$ determines univocally $\hat{\boldsymbol{v}}_{3}$ (up to a global phase factor), the orthogonality of the set $\left(\hat{\boldsymbol{\eta}}_{1}, \hat{\boldsymbol{v}}_{2}, \hat{\boldsymbol{v}}_{3}\right)$ in Eq. (27) constitutes a confirmation that the hypothesis $\phi_{3}=\phi_{2}=0$ is correct (see Appendix B) and matches with the symmetry among the expressions for $\hat{\boldsymbol{v}}_{2}\left(\delta_{2}, \chi_{2}, \theta_{2}\right)$ and $\hat{\boldsymbol{v}}_{3}\left(\delta_{3}, \chi_{3}, \theta_{3}\right)$ in Eq. (27). Different pairs of states $\left(\hat{\boldsymbol{v}}_{2}, \hat{\boldsymbol{v}}_{3}\right)$ are obtained for each value of the elevation angle $\theta_{2}$ of $\hat{\boldsymbol{v}}_{2}$, as illustrated in Fig. 5.

By taking the ratio between the first and third components of $\hat{\boldsymbol{v}}_{3}$ in Eq. (27), we find that the elevation angles $\theta_{2}$ and $\theta_{3}$ for each pair $\left(\hat{\boldsymbol{v}}_{2}, \hat{\boldsymbol{v}}_{3}\right)$ are linked via the ellipticity angle $\chi_{1}$ of $\hat{\boldsymbol{\eta}}_{1}$ as

$$
\cot \theta_{3}=-\tan \theta_{2} \sin ^{2} \chi_{1} .
$$

Thus, in general, the planes $\Pi_{2}$ and $\Pi_{3}$ containing the respective polarization ellipses of $\hat{\boldsymbol{v}}_{2}$ and $\hat{\boldsymbol{v}}_{3}$ are not mutually orthogonal, while both $\Pi_{2}$ and $\Pi_{3}$ are orthogonal to the plane determined by the axes $Z_{1}$ (normal to the polarization ellipse 

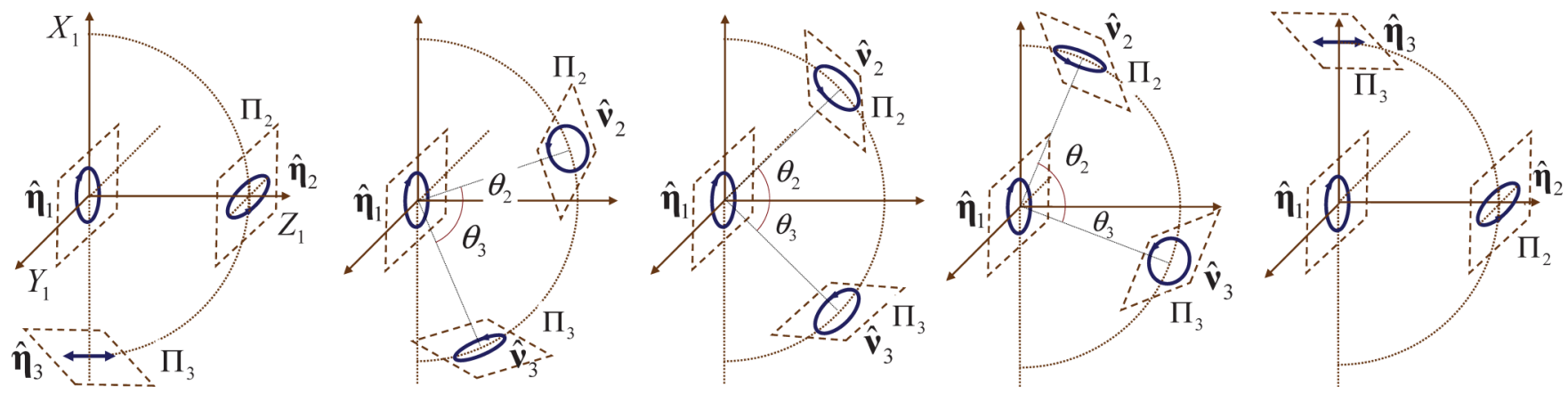

FIG. 5. Physical interpretation of the family of sets of mutually orthogonal states corresponding to $\phi_{2}=\phi_{3}=0$. Although all the states correspond to a common fixed point in space, for the sake of clarity they are represented separated. The planes containing the polarization ellipses of $\hat{\mathbf{v}}_{2}$ and $\hat{\mathbf{v}}_{3}$ are tangent to the semicircle determined by $\phi_{2}$, and perpendicular to the plane $X_{1} Z_{1}$, while $\theta_{2}$ and $\theta_{3}$ are the respective elevation angles about the axis $Y_{1}$.

of $\hat{\boldsymbol{\eta}}_{1}$ ) and $Z_{2}$ (normal to the polarization ellipse of $\hat{\boldsymbol{v}}_{2}$ ). Planes $\Pi_{2}$ and $\Pi_{3}$ reach a symmetric position with respect to the reference axis $Z_{1}$ when $\theta_{3}=-\theta_{2}$, which according to Eq. (29) holds when $\theta_{2}=-\theta_{3}= \pm \arctan \left(1 / \sin \chi_{1}\right)$.

As discussed in Sec. III [Eq. (15) and Fig. 4], for this family (a) of sets of mutually orthonormal states, there exist certain values of $\theta_{i}(i=2,3)$ for which the state $\hat{v}_{i}$ has circular polarization. Regarding $\hat{\mathbf{v}}_{2}$, such circularly polarized states are reached when $\cos \theta_{2}=\mp \tan \chi_{1}$; i.e.,

$$
\begin{aligned}
\hat{\mathbf{v}}_{2}= & e^{i \delta_{2}} \frac{1}{\sqrt{2}}\left(\begin{array}{c}
\mp i \cos \theta_{2} \\
1 \\
\pm i \sin \theta_{2}
\end{array}\right) \\
= & e^{i \delta_{2}} \frac{1}{\sqrt{2}}\left(\begin{array}{c}
i \tan \chi_{1} \\
1 \\
\pm i \sqrt{1-\tan ^{2} \chi_{1}}
\end{array}\right), \\
& \text { with }\left\{\begin{array}{l}
\cos \theta_{2}=\mp \tan \chi_{1} \\
-\pi / 4 \leqslant \chi_{1} \leqslant \pi / 4
\end{array}\right.
\end{aligned}
$$

Thus, for the family of states $\hat{v}_{2}$ with $\phi_{2}=0$, the above circularly polarized states (having unspecified value of $\varphi_{2}$ ) establish the connections between the different branches of $\varphi_{2}=\pi / 2\left(\cos \theta_{2}>\left|\tan \chi_{1}\right|\right)$ and $\varphi_{2}=0\left(\cos \theta_{2}<\left|\tan \chi_{1}\right|\right)$.

As shown in Fig. 4 , the case $\phi_{i}=\pi$ is entirely symmetric with that of $\phi_{i}=0$.

(b) Family of states orthonormal to $\hat{\boldsymbol{\eta}}_{1}$ and with $\phi_{2}=\pi / 2$.

In this case, leaving aside the canonical set $\left(\theta_{i}=0, \pm \pi / 2\right)$ and using the hypothesis $\phi_{3}=\phi_{2}$, Eq. (17) yields

$$
\begin{gathered}
\phi_{3}=\pi / 2, \quad \varphi_{i}=0, \\
\chi_{i}=\arctan \left(-\tan \chi_{1} \cos \theta_{i}\right) .
\end{gathered}
$$

These angular parameters correspond to $\hat{\boldsymbol{v}}_{2}$ and $\hat{\boldsymbol{v}}_{3}$ of the following set of orthonormal polarization states (given as a function of $\chi_{1}$ and the elevation angle $\theta_{2}$ chosen for $\left.\hat{v}_{2}\right)$ :

$$
\begin{aligned}
& \hat{\boldsymbol{\eta}}_{1}=e^{i \delta_{1}}\left(\begin{array}{c}
c_{\chi_{1}} \\
i s_{\chi_{1}} \\
0
\end{array}\right), \\
& \hat{\mathbf{v}}_{2}=e^{i \delta_{2}}\left(\begin{array}{c}
i s_{\chi_{2}} \\
-c_{\chi_{2}} c_{\theta_{2}} \\
-c_{\chi_{2}} s_{\theta_{2}}
\end{array}\right)=\frac{e^{i \delta_{2}}}{\sqrt{1+c_{\chi_{1}}^{2} t_{\theta_{2}}^{2}}}\left(\begin{array}{c}
i s_{\chi_{1}} \\
c_{\chi_{1}} \\
c_{\chi_{1}} t_{\theta_{2}}
\end{array}\right),
\end{aligned}
$$$$
\text { (with } \phi_{2}=\pi / 2, \varphi_{2}=0 \text { ) }
$$

$$
\begin{aligned}
& \hat{\boldsymbol{v}}_{3}=e^{i \delta_{3}}\left(\begin{array}{c}
i s_{\chi_{3}} \\
-c_{\chi_{3}} c_{\theta_{3}} \\
-c_{\chi_{3}} s_{\theta_{3}}
\end{array}\right)=\frac{e^{i \delta_{3}}}{\sqrt{1+c_{\chi_{1}}^{2} t_{\theta_{2}}^{2}}}\left(\begin{array}{c}
-i c_{\chi_{1}} s_{\chi_{1}} t_{\theta_{2}} \\
-c_{\chi_{1}}^{2} t_{\theta_{2}} \\
1
\end{array}\right) \text {, } \\
& \text { (with } \phi_{3}=\pi / 2, \varphi_{3}=0 \text { ). }
\end{aligned}
$$

Note that the uniqueness (up to a global phase factor) of $\hat{v}_{3}$ as a unit vector orthonormal to the pair $\left(\hat{\boldsymbol{\eta}}_{1}, \hat{\boldsymbol{v}}_{2}\right)$, together with the expression of $\hat{\mathbf{v}}_{3}$ in Eq. (32), demonstrates that necessarily $\phi_{3}=\pi / 2$ when $\phi_{2}=\pi / 2$. As occurs for any fixed value of $\phi_{2}$, different pairs of states $\left(\hat{v}_{2}, \hat{v}_{3}\right)$ are obtained for each value of the elevation angle $\theta_{2}$ of $\hat{\boldsymbol{v}}_{2}$, with those corresponding to $\phi_{2}=\pi / 2$ illustrated in Fig. 6 .

Given a particular ellipticity angle $\chi_{1}$ of the first canonical state $\hat{\boldsymbol{\eta}}_{1}$, the respective elevation angles $\theta_{2}$ and $\theta_{3}$ for each pair $\left(\hat{v}_{2}, \hat{v}_{3}\right)$ are connected through the relation

$$
\cot \theta_{3}=-\tan \theta_{2} \cos ^{2} \chi_{1}
$$

As occurs in case (a), the planes $\Pi_{2}$ and $\Pi_{3}$ containing the polarization ellipses of $\hat{\boldsymbol{v}}_{2}$ and $\hat{\boldsymbol{v}}_{3}$ are not mutually orthogonal in general, while both $\Pi_{2}$ and $\Pi_{3}$ are orthogonal to the plane determined by the axes $Z_{1}$ (normal to the polarization ellipse of $\hat{\boldsymbol{\eta}}_{1}$ ) and $Z_{2}$ (normal to the polarization ellipse of $\hat{\boldsymbol{v}}_{2}$ ). According to Eq. (33), planes $\Pi_{2}$ and $\Pi_{3}$ reach a symmetric position with respect to the reference axis $Z_{1}$ when $\theta_{2}=$ $-\theta_{3}= \pm \arctan \left(1 / \cos \chi_{1}\right)$.

Contrary to case (a), and as discussed in Sec. III [Eq. (17) and Fig. 4], the set family (b) of mutually orthonormal states does not contain circularly polarized states, except the particular case where the reference canonical state $\hat{\boldsymbol{\eta}}_{1}$ has circular polarization $\left[\chi_{1}= \pm \pi / 4 \Rightarrow \chi_{2}\left(\eta_{2}\right)=\mp \pi / 4\right]$, but instead $\hat{v}_{i}$ decreases monotonically toward the linear canonical state $\hat{\eta}_{3}$ $\left(\theta_{i}= \pm \pi / 2\right)$ as $\left|\theta_{i}\right|$ increases.

Moreover, orthonormal sets of linearly polarized states can be generated within the family (b) by fixing zero ellipticity for $\hat{\boldsymbol{\eta}}_{1}\left(\chi_{1}=0\right)$, so that $\hat{\boldsymbol{v}}_{2}$ and $\hat{\boldsymbol{v}}_{3}$ have necessarily linear polarizations along mutually orthogonal directions in the plane $Y_{1} Z_{1}$, and the expressions for this particular kind of linearly polarized sets are given by Eq. (23).

As shown in Fig. 4, the case $\phi_{i}=\pi$ is entirely symmetric to that of $\phi_{i}=0$.

Like in the cases $\phi_{2}=0$ (a) and $\phi_{2}=\pi / 2$ (b), any pair $\left(\hat{\mathbf{v}}_{2}, \hat{\boldsymbol{v}}_{3}\right)$ of the orthonormal set $\left(\hat{\boldsymbol{\eta}}_{1}, \hat{\mathbf{v}}_{2}, \hat{\mathbf{v}}_{3}\right)$ always shares a 

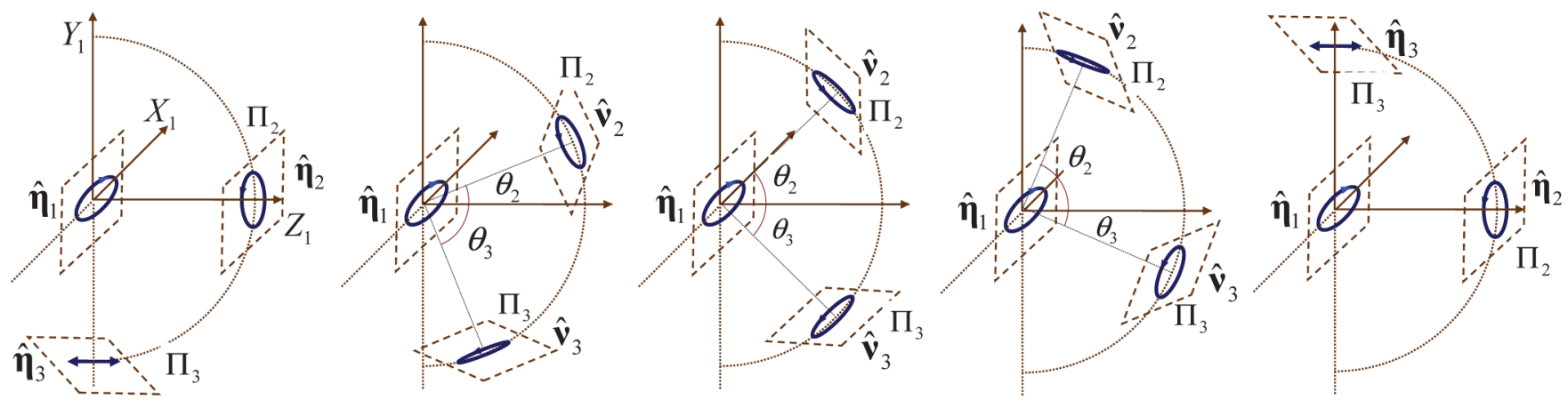

FIG. 6. Physical interpretation of the family of sets of mutually orthogonal states corresponding to $\phi_{2}=\phi_{3}=\pi / 2$. Although all the states correspond to the same point in space, for the sake of clarity they are represented separated. The planes containing the polarization ellipses of $\hat{\mathbf{v}}_{2}$ and $\hat{\boldsymbol{v}}_{3}$ are tangent to the semicircle determined by $\phi_{2}=\phi_{3}=\pi / 2$, and perpendicular to the plane $Y_{1} Z_{1}$, while $\theta_{2}$ and $\theta_{3}$ are the respective elevations about the axis $X_{1}$.

common value for the overall azimuth $\left(\phi_{3}=\phi_{2}\right)$, while they have respective elevation angles with opposite sign (with $\theta_{2}=$ $0, \theta_{3}= \pm \pi / 2$ for the limiting case of the canonical set). Thus, the planes $\Pi_{1}, \Pi_{2}, \Pi_{3}$ containing the respective polarization ellipses of $\hat{\boldsymbol{\eta}}_{1}, \hat{\boldsymbol{v}}_{2}, \hat{\boldsymbol{v}}_{3}$ are all orthogonal to a common plane $\Pi_{\perp}$, and since the spin vector of a given pure state is normal to the plane containing its polarization ellipse, the spin vectors of any orthonormal set $\left(\hat{\boldsymbol{\eta}}_{1}, \hat{\boldsymbol{v}}_{2}, \hat{\boldsymbol{v}}_{3}\right)$ lie in the plane $\Pi_{\perp}$. Furthermore, the electric fields of the states $\left(\hat{\boldsymbol{v}}_{2}, \hat{\boldsymbol{v}}_{3}\right)$ vibrate in planes that, in general, are not orthogonal to the polarizationellipse plane $X_{1} Y_{1}$ of the state $\hat{\boldsymbol{\eta}}_{1}$. According to Eq. (23), the sets of three orthogonal linearly polarized states constitute the only exception to this rule.

\section{INCOHERENT MIXTURE OF TWO CIRCULARLY POLARIZED STATES WHOSE POLARIZATION CIRCLES LIE IN MUTUALLY ORTHOGONAL PLANES}

To illustrate the powerful features and the applicability of the approach presented for the geometric and physical interpretation of sets of orthonormal 3D Jones vectors, let us consider the incoherent composition of two right-circularlypolarized states with the same intensity $I=1 / 2$ and whose respective polarization ellipses lie in mutually orthogonal planes. It seems intuitive that the Jones vectors of these states are orthogonal, but the spectral decomposition performed below, combined with the formalism introduced in this work, shows that the eigenstates of this incoherent composition are substantially different from those of the starting components.

By taking the reference axes $X$ and $Y$ to be orthogonal to the polarization-circle planes of the two components, the polarization density matrix of the composed state becomes

$$
\begin{aligned}
\hat{\mathbf{R}} & =\frac{1}{2} \hat{\mathbf{R}}_{\mathrm{cx}}+\frac{1}{2} \hat{\mathbf{R}}_{\mathrm{cy}} \\
& =\frac{1}{2}\left(\begin{array}{ccc}
0 & 0 & 0 \\
0 & 1 / 2 & i / 2 \\
0 & -i / 2 & 1 / 2
\end{array}\right)+\frac{1}{2}\left(\begin{array}{ccc}
1 / 2 & 0 & i / 2 \\
0 & 0 & 0 \\
-i / 2 & 0 & 1 / 2
\end{array}\right) \\
& =\frac{1}{4}\left(\begin{array}{ccc}
1 & 0 & i \\
0 & 1 & i \\
-i & -i & 2
\end{array}\right) .
\end{aligned}
$$

Since this state has been built as an incoherent composition of two pure states, it follows that rank $\hat{\mathbf{R}}=2$, and its eigenvalues are

$$
\hat{\lambda}_{1}=3 / 4, \quad \hat{\lambda}_{2}=1 / 4, \quad \hat{\lambda}_{3}=0,
$$

with respective eigenstates

$$
\begin{aligned}
& \hat{\mathbf{u}}_{1}=\frac{1}{\sqrt{6}}(1,1,-2 i)^{\mathrm{T}}, \quad \hat{\mathbf{u}}_{2}=\frac{1}{\sqrt{2}}(1,-1,0)^{\mathrm{T}}, \\
& \hat{\mathbf{u}}_{3}=\frac{1}{\sqrt{3}}(1,1, i)^{\mathrm{T}} .
\end{aligned}
$$

The next step is to transform $\hat{\mathbf{u}}_{1}$ into its representative form $\hat{\eta}_{1}$ with respect to its own intrinsic reference frame $X_{1} Y_{1} Z_{1}$, which is realized through the transformation

$$
\begin{gathered}
\hat{\boldsymbol{\eta}}_{1}=e^{i \pi / 2} \mathbf{Q}^{\mathrm{T}} \hat{\mathbf{u}}_{1}=\left(\begin{array}{c}
\sqrt{2 / 3} \\
i / \sqrt{3} \\
0
\end{array}\right), \\
\mathbf{Q}^{\mathrm{T}}=\left(\begin{array}{ccc}
0 & 0 & 1 \\
1 / \sqrt{2} & 1 / \sqrt{2} & 0 \\
1 / \sqrt{2} & -1 / \sqrt{2} & 0
\end{array}\right),
\end{gathered}
$$

where the rotation matrix $\mathbf{Q}$ can be obtained via the procedure shown in [31]. Note that the ellipticity angle $\chi_{1}$ of $\hat{\eta}_{1}$ (and of $\hat{\mathbf{u}}_{1}$, because of the invariance of $\chi_{1}$ with respect to rotations of the coordinate system) has the value $\chi_{1}=\arctan (1 / \sqrt{2}) \approx$ $35.26^{\circ}$, thus corresponding to a noncircular state.

Therefore, the set of eigenstates of $\hat{\mathbf{R}}$, when represented with respect to the coordinate system $X_{1} Y_{1} Z_{1}$ (by applying rotation $\mathbf{Q}^{\mathrm{T}}$ ), takes the form

$$
\hat{\boldsymbol{\eta}}_{1}=\left(\begin{array}{c}
\sqrt{2 / 3} \\
i / \sqrt{3} \\
0
\end{array}\right), \hat{\mathbf{v}}_{2}=\left(\begin{array}{l}
0 \\
0 \\
1
\end{array}\right), \hat{\mathbf{v}}_{3}=\left(\begin{array}{c}
i / \sqrt{3} \\
\sqrt{2 / 3} \\
0
\end{array}\right),
$$

which due to the symmetry of the starting composition incidentally coincide with a canonical set (with $\hat{\mathbf{v}}_{2}=\hat{\mathbf{\eta}}_{3}, \hat{\mathbf{v}}_{3}=$ $\hat{\boldsymbol{\eta}}_{2}$ ). Consequently, the polarization density matrix $\hat{\mathbf{R}}_{1}$, representing the same original state $\hat{\mathbf{R}}$ but in the intrinsic reference 
frame $X_{1} Y_{1} Z_{1}$ of $\hat{\mathbf{u}}_{1}$, has the spectral structure

$$
\hat{\mathbf{R}}_{1}=\mathbf{Q}^{\mathrm{T}} \hat{\mathbf{R}} \mathbf{Q}=\frac{3}{4}\left(\hat{\boldsymbol{\eta}}_{1} \otimes \hat{\boldsymbol{\eta}}_{1}^{\dagger}\right)+\frac{1}{4}\left(\hat{\boldsymbol{\eta}}_{3} \otimes \hat{\boldsymbol{\eta}}_{3}^{\dagger}\right) .
$$

Thus, in view of the formalism of this work, $\hat{\mathbf{R}}_{1}$ can be viewed as an incoherent composition of an elliptically polarized state in the $X_{1} Y_{1}$ plane $\left(\hat{\eta}_{1}\right)$, with ellipticity angle $\chi_{1}=\arctan (1 / \sqrt{2})$, and a linearly polarized state along the axis $Z_{1}\left(\hat{\eta}_{3}\right)$, having respective relative weights (intensities) $3 / 4$ and $1 / 4$.

From Eq. (3) we find that the characteristic decomposition of $\hat{\mathbf{R}}$ in the original frame is given by

$$
\begin{aligned}
\hat{\mathbf{R}} & =P_{1} \hat{\mathbf{R}}_{p}+\left(1-P_{1}\right) \hat{\mathbf{R}}_{m}, \quad\left(P_{1}=1 / 2\right), \\
\hat{\mathbf{R}}_{p} & \equiv \hat{\mathbf{u}}_{1} \otimes \hat{\mathbf{u}}_{1}^{\dagger}=\frac{1}{6}\left(\begin{array}{ccc}
1 & 1 & 2 i \\
1 & 1 & 2 i \\
-2 i & -2 i & 4
\end{array}\right), \\
\hat{\mathbf{R}}_{m} & \equiv \frac{1}{2}\left[\left(\hat{\mathbf{u}}_{1} \otimes \hat{\mathbf{u}}_{1}^{\dagger}\right)+\left(\hat{\mathbf{u}}_{2} \otimes \hat{\mathbf{u}}_{2}^{\dagger}\right)\right] \\
& =\frac{1}{6}\left(\begin{array}{ccc}
2 & -1 & i \\
-1 & 2 & i \\
-i & -i & 2
\end{array}\right),
\end{aligned}
$$

whereas the same decomposition of $\hat{\mathbf{R}}_{1}$ in the reference frame $X_{1} Y_{1} Z_{1}$ reads

$$
\begin{aligned}
\hat{\mathbf{R}}_{1} & =P_{1} \hat{\mathbf{R}}_{p 1}+\left(1-P_{1}\right) \hat{\mathbf{R}}_{m 1}, \quad\left(P_{1}=1 / 2\right), \\
\hat{\mathbf{R}}_{p 1} & \equiv \hat{\boldsymbol{\eta}}_{1} \otimes \hat{\boldsymbol{\eta}}_{1}^{\dagger}=\frac{1}{3}\left(\begin{array}{ccc}
2 & -i \sqrt{2} & 0 \\
i \sqrt{2} & 1 & 0 \\
0 & 0 & 0
\end{array}\right), \\
\hat{\mathbf{R}}_{m 1} & \equiv \frac{1}{2}\left[\left(\hat{\boldsymbol{\eta}}_{1} \otimes \hat{\boldsymbol{\eta}}_{1}^{\dagger}\right)+\left(\hat{\boldsymbol{\eta}}_{3} \otimes \hat{\boldsymbol{\eta}}_{3}^{\dagger}\right)\right] \\
& =\frac{1}{6}\left(\begin{array}{ccc}
2 & -i \sqrt{2} & 0 \\
i \sqrt{2} & 1 & 0 \\
0 & 0 & 3
\end{array}\right)
\end{aligned}
$$

The pure characteristic component coincides (as always) with the spectral component that has the largest eigenvalue, but with coefficient $P_{1}=1 / 2$ instead of the coefficient $\hat{\lambda}_{1}=3 / 4$ corresponding to the spectral decomposition. Since $\hat{\mathbf{R}}_{m 1}$ is a complex matrix, it represents a nonregular state [26,29], and the theoretical framework put forward in this work allows one to interpret the component $\hat{\mathbf{R}}_{m 1}$, which determines the regularity of $\hat{\mathbf{R}}_{1}$ (and $\mathbf{R}$ ), as an equiprobable mixture of the noncoplanar states $\hat{\eta}_{1}$ and $\hat{\eta}_{3}$.

This example illustrates how the identification of the geometric characteristics of the spectral components of a given partially polarized 3D state enhances its physical interpretation and provides meaningful analyses.

\section{CONCLUSIONS}

The algebraic formulation of the properties of sets of three orthonormal 3D Jones vectors has provided a meaningful geometric representation of such states, which play an essential role for the physical understanding and interpretation of both the spectral and the characteristic decompositions of the polarization matrix of any given $3 \mathrm{D}$ partially polarized state.

The inspection of the results obtained in the previous sections allows us to summarize them through the following conclusions:

(1) Given a pure polarization state $\hat{\eta}_{1}$ and an arbitrary plane $\Pi_{2}$, it is always possible to find a pure state $\hat{v}_{2}$ orthogonal to $\hat{\eta}_{1}$ whose polarization ellipse lies in $\Pi_{2}$. The azimuth $\varphi_{2}$ and ellipticity angle $\chi_{2}$ of $\hat{v}_{2}$ depend on the ellipticity angle $\chi_{1}$ of $\hat{\eta}_{1}$ and on the angles $\phi_{2}$ and $\theta_{2}$ determining the plane $\Pi_{2}$. Given $\hat{\eta}_{1}$, and once $\Pi_{2}$ is specified, the plane containing the polarization ellipse of the third orthonormal state $\hat{v}_{3}$ is fully determined, together with its azimuth $\varphi_{3}$ and ellipticity angle $\chi_{3}$.

(2) Given a pure polarization state $\hat{\eta}_{1}$, any pure states $\hat{\boldsymbol{v}}_{2}$ and $\hat{\boldsymbol{v}}_{3}$ that together with $\hat{\eta}_{1}$ constitute an orthonormal set exhibit necessarily a common value for the overall azimuth, i.e., $\phi_{3}=\phi_{2}$, while $\hat{\mathbf{v}}_{2}$ and $\hat{\mathbf{v}}_{3}$ have respective elevation angles with opposite signs, viz., $\left(\theta_{3} /\left|\theta_{3}\right|=-\theta_{2} /\left|\theta_{2}\right|\right)$. For the particular case of the canonical set, $\theta_{2}=0$ and $\theta_{3}= \pm \pi / 2$.

(3) Once the state $\hat{\eta}_{1}$ with its polarization ellipse in the $X_{1} Y_{1}$ plane is taken as reference, the electric fields of the complementary pair $\left(\hat{\mathbf{v}}_{2}, \hat{\mathbf{v}}_{3}\right)$ vibrate in planes that are not orthogonal to $X_{1} Y_{1}$, except for the case of sets of linearly polarized states. Note that the respective planes of the canonical pair $\left(\hat{\boldsymbol{\eta}}_{1}, \hat{\boldsymbol{\eta}}_{3}\right)$ are mutually orthogonal, and the same concerns the pair $\left(\hat{\eta}_{2}, \hat{\eta}_{3}\right)$.

(4) As shown in Sec. IV, families of three orthonormal linearly polarized states necessarily satisfy $\phi_{2}=\phi_{3}= \pm \pi / 2$, while their elevation angles form a right angle, $\left|\theta_{2}-\theta_{3}\right|=$ $\pi / 2$. This is the only case where the polarization ellipses of the components of the orthonormal set $\left(\hat{\boldsymbol{\eta}}_{1}, \hat{\mathbf{v}}_{2}, \hat{\mathbf{v}}_{3}\right)$ lie in respective planes that are mutually orthogonal.

(5) Since $\phi_{2}=\phi_{3}$, together with the fact that the spin vector of a given pure state is normal to the plane containing its polarization ellipse, the respective spin vectors of any set of orthogonal polarization states lie in a common plane that is orthogonal to the associated planes containing the polarization ellipses of such states.

(6) A meaningful physical interpretation of the spectral decomposition of a given polarization matrix $\mathbf{R}$ is achieved by taking the first eigenstate $\hat{\mathbf{u}}_{1}$ (i.e., the eigenvector of $\mathbf{R}$ with the largest eigenvalue $\lambda_{1}$ ) as the reference vector $\hat{\eta}_{1}$, so that the unit eigenstates become the corresponding set ( $\hat{\boldsymbol{\eta}}_{1}, \hat{\mathbf{v}}_{2}, \hat{\mathbf{v}}_{3}$ ). Then the polarization matrix $\mathbf{R}_{1}$, describing the same state $\mathbf{R}$ but in the intrinsic reference frame of $\hat{\mathbf{u}}_{1}$, can be considered as an incoherent superposition (at the point $\mathbf{r}$ in space considered) of the states $\left(\hat{\boldsymbol{\eta}}_{1}, \hat{\mathbf{v}}_{2}, \hat{\mathbf{v}}_{3}\right)$, i.e.,

$$
\mathbf{R}_{1}=I\left[\hat{\lambda}_{1}\left(\hat{\boldsymbol{\eta}}_{1} \otimes \hat{\boldsymbol{\eta}}_{1}^{\dagger}\right)+\hat{\lambda}_{2}\left(\hat{\mathbf{v}}_{2} \otimes \hat{\mathbf{v}}_{2}^{\dagger}\right)+\hat{\lambda}_{3}\left(\hat{\mathbf{v}}_{3} \otimes \hat{\mathbf{v}}_{3}^{\dagger}\right)\right]
$$

where the normalized eigenvalues $\left(\hat{\lambda}_{1}, \hat{\lambda}_{2}, \hat{\lambda}_{3}\right)$ are the respective weights and $I=\operatorname{tr} \mathbf{R}_{O}=\operatorname{tr} \mathbf{R}$ is the intensity of the state. The transformation from $\mathbf{R}$ to $\mathbf{R}_{1}$ can be performed through the procedure indicated in Ref. [31].

(7) The characteristic decomposition of a given polarization matrix $\mathbf{R}$ can also be interpreted in a privileged form when $\hat{\mathbf{u}}_{1}$ is taken as $\hat{\eta}_{1}$, in which case the polarization state 
can be expressed as

$$
\begin{aligned}
\mathbf{R}_{1} & =P_{1} \mathbf{R}_{p}+\left(1-P_{1}\right) \mathbf{R}_{m}+\left(P_{2}-P_{1}\right) \mathbf{R}_{u-3 \mathrm{D}}, \\
\mathbf{R}_{p} & \equiv I\left(\hat{\boldsymbol{\eta}}_{1} \otimes \hat{\boldsymbol{\eta}}_{1}^{\dagger}\right), \\
\mathbf{R}_{m} & \equiv I \frac{1}{2}\left[\left(\hat{\boldsymbol{\eta}}_{1} \otimes \hat{\boldsymbol{\eta}}_{1}^{\dagger}\right)+\left(\hat{\boldsymbol{v}}_{2} \otimes \hat{\mathbf{v}}_{2}^{\dagger}\right)\right], \\
\mathbf{R}_{u-3 \mathrm{D}} & \equiv I \frac{1}{3} \operatorname{diag}(1,1,1),
\end{aligned}
$$

where $\mathbf{R}_{p}$ represents the characteristic pure state determined by $\hat{\boldsymbol{\eta}}_{1}, \mathbf{R}_{u-3 \mathrm{D}}$ represents a fully random $3 \mathrm{D}$ state, and the middle state $\mathbf{R}_{m}$ is given by an equiprobable mixture of the sates $\hat{\eta}_{1}$ and $\hat{\boldsymbol{v}}_{2}$. Note that the pair $\left(\hat{\boldsymbol{\eta}}_{1}, \hat{\mathbf{v}}_{2}\right)$ is sufficient for a simple and meaningful physical interpretation of $\mathbf{R}$ in terms of the characteristic decomposition. The particular properties of the pair $\left(\hat{\boldsymbol{\eta}}_{1}, \hat{\mathbf{v}}_{2}\right)$ determine the regularity [26,29] of the state $\mathbf{R}$ under consideration.

In summary, the results obtained in this work provide a powerful tool for the analysis and physical understanding of three-dimensional partially polarized states. Beyond polarization phenomena, our framework provides a general and complete geometric interpretation of $3 \times 3$ unitary matrices, with potential applications in areas where such matrices play a key role, for instance, three-level quantum systems and gates for ternary quantum logic circuits.

\section{ACKNOWLEDGMENTS}

The authors acknowledge the Academy of Finland (Grants No. 308393 and No. 310511); the Swedish Cultural Foundation in Finland (A.N.); Joensuu University Foundation (J.J.G.). The work is part of the Academy of Finland Flagship Programme, Photonics Research and Innovation (PREIN, 320166).

\section{APPENDIX A: DETERMINATION OF THE ANGULAR PARAMETERS OF A GIVEN 3D JONES VECTOR}

Let us consider the inverse problem of obtaining the angular parameters $(\theta, \phi, \varphi, \chi)$ of a given unit Jones vector $\hat{\varepsilon}$. To do so, we take as known data the complex-valued components of $\hat{\varepsilon}$, whose real and imaginary parts are denoted as

$$
\hat{\varepsilon}=\left(a_{x}+i b_{x}, a_{y}+i b_{y}, a_{z}+i b_{z}\right)^{\mathrm{T}} .
$$

Then, on using Eqs. (7) and (8), the angular parameters (except for the phase factor $\gamma$ ) are fully determined, in their corresponding quadrants, in terms of $a_{i}$ and $b_{i}$ via the following expressions:

$$
\begin{aligned}
& \tan ^{2} \chi=\frac{b_{x}^{2}+b_{y}^{2}+b_{z}^{2}}{a_{x}^{2}+a_{y}^{2}+a_{z}^{2}}, \quad\left\{\begin{array}{l}
-\pi / 4 \leqslant \chi \leqslant \pi / 4, \\
\operatorname{sgn}(\chi)=\operatorname{sgn}\left(a_{x} b_{y}-a_{y} b_{x}\right),
\end{array}\right. \\
& \tan \varphi=-\frac{1}{\tan \chi} \frac{b_{z}}{a_{z}}, \quad(0 \leqslant \varphi<\pi) \\
& \tan \phi=\frac{a_{x} b_{z}-a_{z} b_{x}}{a_{y} b_{z}-a_{z} b_{y}}, \quad(-\pi<\phi \leqslant \pi), \\
& \operatorname{sgn}(\sin \phi)=\operatorname{sgn}\left(-a_{y} \frac{\cos \varphi}{\cos \chi}+b_{y} \frac{\sin \varphi}{\sin \chi}\right) \text {, }
\end{aligned}
$$

$\operatorname{sgn}(\cos \phi)=\operatorname{sgn}\left(a_{x} \frac{\cos \varphi}{\cos \chi}-b_{x} \frac{\sin \varphi}{\sin \chi}\right)$,

where sgn stands for the sign function.

\section{APPENDIX B: ON THE OVERALL AZIMUTH OF THE COMPONENTS $\hat{v}_{2}$ AND $\hat{v}_{3}$ OF THE ORTHONORMAL SET OF 3D JONES VECTORS $\left(\hat{\eta}_{1}, \hat{v}_{2}, \hat{v}_{3}\right)$}

Let us consider an arbitrary orthonormal set of 3D Jones vectors $\left(\hat{\boldsymbol{\eta}}_{1}, \hat{\boldsymbol{v}}_{2}, \hat{\mathbf{v}}_{3}\right)$ represented, as in Sec. III, with respect to the intrinsic reference frame $X_{1} Y_{1} Z_{1}$ of $\hat{\eta}_{1}$. This section is devoted to demonstrate that the equality $\left|\phi_{3}\right|=\left|\phi_{2}\right|$ is always satisfied, where $\phi_{3}$ and $\phi_{2}$ are the overall azimuths of $\hat{v}_{3}$ and $\hat{\mathbf{v}}_{2}$, respectively.

We first recall that the general expression of a $3 \times 3$ unitary matrix, whose first column is constituted by $\hat{\eta}_{1}$, is given by (see Sec. 4 of [31])

$$
\mathbf{V}_{1}=\left(\begin{array}{ccc}
e^{i \alpha_{1}} \cos \chi_{1} & i e^{i \alpha_{2}} \cos \mu \sin \chi_{1} & i e^{i \alpha_{3}} \sin \mu \sin \chi_{1} \\
i e^{i \alpha_{1}} \sin \chi_{1} & e^{i \alpha_{2}} \cos \mu \cos \chi_{1} & e^{i \alpha_{3}} \sin \mu \cos \chi_{1} \\
0 & e^{i \beta_{2}} \sin \mu & -e^{i\left(\beta_{2}-\alpha_{2}+\alpha_{3}\right)} \cos \mu
\end{array}\right)
$$

Columns 2 and 3 of $\mathbf{V}_{1}$ are precisely the vectors $\hat{\mathbf{v}}_{2}$ and $\hat{\mathbf{v}}_{3}$ written as functions of the ellipticity angle $\chi_{1}$ of $\hat{\eta}_{1}$ and the auxiliary angles $\mu, \alpha_{2}, \alpha_{3}, \beta_{2}$. Thus, according to Eq. (11), by expressing the components of $\hat{\boldsymbol{v}}_{2}$ and $\hat{\mathbf{v}}_{3}$ in terms of their real and imaginary parts we get

$$
\begin{aligned}
& t_{\chi_{i}}=t_{\chi_{1}} \frac{t_{\varphi_{i}}-c_{\theta_{i}} t_{\phi_{i}}}{t_{\phi_{i}}-c_{\theta_{i}} t_{\varphi_{i}}}, \\
& \hat{\mathbf{v}}_{2}=\left(\begin{array}{c}
-s_{\alpha_{2}} c_{\mu} s_{\chi_{1}}+i c_{\alpha_{2}} c_{\mu} s_{\chi_{1}} \\
c_{\alpha_{2}} c_{\mu} c_{\chi_{1}}+i s_{\alpha_{2}} c_{\mu} c_{\chi_{1}} \\
c_{\beta_{2}} s_{\mu}+i s_{\beta_{2}} s_{\mu}
\end{array}\right), \\
& \hat{\mathbf{v}}_{3}=\left(\begin{array}{c}
-s_{\alpha_{3}} s_{\mu} s_{\chi_{1}}+i c_{\alpha_{3}} s_{\mu} s_{\chi_{1}} \\
c_{\alpha_{3}} s_{\mu} c_{\chi_{1}}+i s_{\alpha_{3}} s_{\mu} c_{\chi_{1}} \\
-c_{\beta_{3}} c_{\mu}-i s_{\beta_{3}} c_{\mu}
\end{array}\right), \\
&\left(\beta_{3}=\beta_{2}-\alpha_{2}+\alpha_{3}\right) .
\end{aligned}
$$

The angles $\phi_{2}$ and $\phi_{3}$ can now be calculated by using Eq. (A2), which leads to

$$
\tan \phi_{2}=\tan \phi_{3}=\tan \chi_{1} \cot \left(\alpha_{2}-\beta_{2}\right),
$$

and shows that $\left|\phi_{3}\right|=\left|\phi_{2}\right|$. Concerning the question whether $\phi_{3}=\phi_{2}$, the nature of Eq. (A2) makes it difficult to carry out a general analytic demonstration. Nevertheless, such equality is demonstrated in Sec. $\mathrm{V}$ for the particular cases $\phi_{2}=0$ and $\phi_{2}=\pi$. Furthermore, numerical calculations made for a series of cases with different values of $\phi_{2}$ are also consistent with $\phi_{3}=\phi_{2}$, justifying its consideration as a general property. 
[1] J. J. Gil, Polarimetric characterization of light and media, Eur. Phys. J.: Appl. Phys. 40, 1 (2007).

[2] J. J. Gil and R. Ossikovski, Polarized Light and the Mueller Matrix Approach (CRC Press, Boca Raton, FL, 2016).

[3] T. Setälä, M. Kaivola, and A. T. Friberg, Degree of Polarization in Near Fields of Thermal Sources: Effects of Surface Waves, Phys. Rev. Lett. 88, 123902 (2002).

[4] T. Setälä, A. Shevchenko, M. Kaivola, and A. T. Friberg, Degree of polarization for optical near fields, Phys. Rev. E 66, 016615 (2002).

[5] M. R. Dennis, Geometric interpretation of the threedimensional coherence matrix for nonparaxial polarization, J. Opt. A: Pure Appl. Opt. 6, S26 (2004).

[6] A. Luis, Degree of polarization for three-dimensional fields as a distance between correlation matrices, Opt. Commun. 253, 10 (2005).

[7] J. C. Petruccelli, N. J. Moore, and M. A. Alonso, Two methods for modeling the propagation of the coherence and polarization properties of nonparaxial fields, Opt. Commun. 283, 4457 (2010).

[8] C. J. R. Sheppard, Partial polarization in three dimensions, J. Opt. Soc. Am. A 28, 2655 (2011).

[9] J. M. Auñón and M. Nieto-Vesperinas, On two definitions of the three-dimensional degree of polarization in the near field of statistically homogeneous partially coherent sources, Opt. Lett. 38, 58 (2013).

[10] O. Gamel and D. F. V. James, Majorization and measures of classical polarization in three dimensions, J. Opt. Soc. Am. A 31, 1620 (2014).

[11] J. J. Gil, Interpretation of the coherency matrix for threedimensional polarization states, Phys. Rev. A 90, 043858 (2014).

[12] J. J. Gil, Components of purity of a three-dimensional polarization state, J. Opt. Soc. Am. A 33, 40 (2016).

[13] A. Norrman, A. T. Friberg, J. J. Gil, and T. Setälä, Dimensionality of random light fields, J. Eur. Opt. Soc. - Rapid Publ. 13, 36 (2017).

[14] K. Lindfors, A. Priimagi, T. Setälä, A. Shevchenko, A. T. Friberg, and M. Kaivola, Local polarization of tightly focused unpolarized light, Nat. Photonics 1, 228 (2007).

[15] T. Bauer, P. Banzer, E. Karimi, S. Orlov, A. Rubano, L. Marrucci, E. Santamato, R. W. Boyd, and G. Leuchs, Observation of optical polarization Möbius strips, Science 347, 964 (2015).
[16] Y. Chen, A. Norrman, S. A. Ponomarenko, and A. T. Friberg, Partially coherent axiconic surface plasmon polariton fields, Phys. Rev. A 97, 041801(R) (2018).

[17] Y. Chen, A. Norrman, S. A. Ponomarenko, and A. T. Friberg, Coherence lattices in surface plasmon polariton fields, Opt. Lett. 43, 3429 (2018).

[18] A. Norrman, T. Setälä, and A. T. Friberg, Partial spatial coherence and partial polarization in random evanescent fields on lossless interfaces, J. Opt. Soc. Am. A 28, 391 (2011).

[19] A. Norrman, T. Setälä, and A. T. Friberg, Generation and electromagnetic coherence of unpolarized three-component light fields, Opt. Lett. 40, 5216 (2015).

[20] R. C. Jones, A new calculus for the treatment of optical systems. I, J. Opt. Soc Am. 31, 488 (1941).

[21] R. M. A. Azzam, Three-dimensional polarization states of monochromatic light fields, J. Opt. Soc. Am. A 28, 2279 (2011).

[22] T. Setälä, A. Shevchenko, M. Kaivola, and A. T. Friberg, Polarization time and length for random optical beams, Phys. Rev. A 78, 033817 (2008).

[23] T. Voipio, T. Setälä, A. Shevchenko, and A. T. Friberg, Polarization dynamics and polarization time of random threedimensional electromagnetic fields, Phys. Rev. A 82, 063807 (2010).

[24] A. Shevchenko, M. Roussey, A. T. Friberg, and T. Setälä, Polarization time of unpolarized light, Optica 4, 64 (2017).

[25] J. J. Gil and I. San José, 3D polarimetric purity, Opt. Commun. 283, 4430 (2010).

[26] J. J. Gil, A. T. Friberg, T. Setälä, and I. San José, Structure of polarimetric purity of three-dimensional polarization states, Phys. Rev. A 95, 053856 (2017).

[27] J. J. Gil, A. Norrman, A. T. Friberg, and T. Setälä, Polarimetric purity and the concept of degree of polarization, Phys. Rev. A 97, 023838 (2018).

[28] I. San José and J. J. Gil, Invariant indices of polarimetric purity. Generalized indices of purity for $n \times n$ covariance matrices, Opt. Commun. 284, 38 (2011).

[29] J. J. Gil, A. Norrman, A. T. Friberg, and T. Setälä, Nonregularity of three-dimensional polarization states, Opt. Lett. 43, 4611 (2018).

[30] A. Norrman, J. J. Gil, A. T. Friberg, and T. Setälä, Polarimetric nonregularity of evanescent waves, Opt. Lett. 44, 215 (2019).

[31] J. J. Gil, Parametrization of $3 \times 3$ unitary matrices based on polarization algebra, Eur. Phys. J. Plus 133, 206 (2018). 